\title{
Ground States of Fermions on Lattices
}

\section{Taku Matsui}

Department of Mathematics, Tokyo Metropolitan University, 1-1 Minami Ohsawa, Hachioji-shi, Tokyo 192-03, Japan E-mail: matsui@math metro-u ac jp

Received: 4 August 1995/Accepted: 2 July 1996

\begin{abstract}
We consider Fermion systems on integer lattices. We establish the existence of dynamics for a class of long range interactions. The infinite volume ground states are considered. The equivalence of the variational principle and ground state conditions is proved for long range interactions. We also prove that any pure translationally invariant ground state of the gauge invariant algebra is extendible to a ground state of the full CAR algebra for the Hamiltonian with a chemical potential (equivalence of ensemble for canonical and ground canonical states at the zero temperature).
\end{abstract}

\section{Introduction}

In this paper we consider lattice Fermion Hamiltonians and their ground states. We consider the infinite volume systems directly by use of functional analytic techniques. The main object of this paper is as follows. (1) We establish the existence of the time evolution as the one-parameter group of automorphisms on the algebra of observables (Heisenberg picture). (2) We show that the infinite volume ground state in the sense of (1.13) below is characterized via the minimization of energy. Equation (1.13) means the positive energy representation while the principle of minimization of energy expectation value is the Gibbs variational principle at zero temperature. (3) Any translationally invariant pure ground state of the gauge invariant algebra can be extended to a ground state of the full CAR algebra for the Hamiltonian with a chemical potential term.

The prototype of the interaction we have in mind is the spinless translationally invariant Hamiltonian $H$,

$$
H=\sum_{\mathbf{Z}^{d} \ni k, l} t_{k l} a_{k}^{*} a_{l}+\sum_{A} W_{A} \prod_{A \ni j} n_{j},
$$

where $a_{k}^{*} a_{l}$ and $n_{J}$ are Fermion creation, annihilation and number operators and $W_{A}$ is a real number (=coupling constant) depending on the finite subset $A$ of $\mathbf{Z}^{d}$. The translational invariance means that $t_{i j}=t_{0 i-j}$ and that $W_{A}=W_{A-j}$. The decay 
condition we will assume is the finiteness of the following numbers:

$$
\sum_{l \in \mathbf{Z}^{d}}\left|t_{k l}\right|<\infty, \quad \sum_{A \ni 0}|A|\left|W_{A}\right|<\infty .
$$

Stronger conditions are often used in [5] and [18]. Part of the results of this article was announced in [14].

We will consider the spinless Fermion on the integer lattice $\mathbf{Z}^{d}$. Except for Theorem 1.5 and Proposition 4.8 the same results are valid when the Fermion field has a finite number of components and the gauge group is a compact Lie group and the lattice has the periodicity.

We use operator algebraic language. For details of the basic notions see [5]. Let us begin with fixing notations. Let $\mathscr{A}$ be the CAR (canonical anti-commutation relation) algebra. $\mathscr{A}$ is the $C^{*}$-algebra with unit generated by $a_{j}^{*}, a_{k}\left(j, k \in \mathbf{Z}^{d}\right)$ satisfying

$$
\left\{a_{j}, a_{k}\right\}=\left\{a_{j}^{*}, a_{k}^{*}\right\}=0, \quad\left\{a_{j}, a_{k}^{*}\right\}=\delta_{j k} 1 .
$$

It is often useful to handle the notation of the Clifford algebra. So we introduce $b_{(j,+)}$ and $b_{(j,-)}$ via the equations,

$$
b_{(j,+)}=a_{j}^{*}+a_{j}, \quad b_{(j,-)}=\frac{a_{j}^{*}-a_{j}}{\sqrt{-1}} .
$$

Thus we have

$$
b_{\alpha}=b_{\alpha}^{*}, \quad\left\{b_{\alpha}, b_{\beta}\right\}=\delta_{\alpha, \beta} 1
$$

for $\alpha$ and $\beta$ in $\Gamma=\mathbf{Z}^{d} \times\{+,-\}$.

Let $j$ be a site in $\mathbf{Z}^{d}$. Let $A=\left\{\alpha_{1}, \alpha_{2}, \ldots, \alpha_{m}\right\}$ be a finite subset of $\Gamma=\mathbf{Z}^{d} \times$ $\{+,-\}\left(\alpha_{k}=\left(l_{k}, \varepsilon_{k}\right), k=1,2, \ldots, m\right.$ with $l_{k} \in \mathbf{Z}^{d}, \varepsilon_{k}= \pm$. $)$ We set $A+j=\left\{\left(l_{k}+\right.\right.$ $\left.\left.j, \varepsilon_{k}\right), k=1,2, \ldots, m\right\}$. By $A \ni j$ we mean $A \ni(j,+)$ or $A \ni(j,-)$ when $A \subset \Gamma$ and $j \in \mathbf{Z}^{d}$.

We fix an order for the elements of $\Gamma$. (For our purpose, any choice of order will do.) Then for a finite subset $\mathrm{A}$ of $\Gamma, A=\left\{\alpha_{1}, \alpha_{2}, \ldots, \alpha_{m}\right\}$ we introduce the ordered product,

$$
b(A)=b_{\alpha_{1}} b_{\alpha_{2}} \cdots b_{\alpha_{m}}=\prod_{A \ni \alpha} b_{\alpha} .
$$

By $\Lambda$ we denote a $d$ dimensional cube and $|\Lambda|$ will be the total number of lattice sites in $\Lambda$. The thermodynamic limit (= the infinite volume limit) is taken for sequences of $d$ dimensional cubes, so $\lim _{\Lambda}$ means that the limit is taken for the sequence of $d$ dimensional cubes $\Lambda$ which converges to $\mathbf{Z}^{d}$. Needless to say, the van Hove limit can be taken without modifying results.

Let $\Lambda$ be a subset of $\mathbf{Z}^{d}$. By $\mathscr{A}_{\Lambda}$ we denote the $C^{*}$-subalgebra generated by $a_{j}^{*}, a_{k}$ with $j, k \in \Lambda$ and $\mathscr{A}_{\text {loc }}=\bigcup_{|\Lambda|<\infty} \mathscr{A}_{\Lambda}$. So an element of $\mathscr{A}_{\text {loc }}$ is an operator supported in a finite subset of $\mathbf{Z}^{d}$. By $\tau_{j}\left(j \in \mathbf{Z}^{d}\right)$, we denote the lattice translation which is an automorphism of $\mathscr{A}$ determined via the equations

$$
\tau_{j}\left(a_{k}\right)=a_{j+k}, \quad \tau_{j}\left(a_{k}^{*}\right)=a_{j+k}^{*} .
$$

Let $\Theta$ be the automorphism of $\mathscr{A}$ determined by $\Theta\left(a_{j}\right)=-a_{j}$ for all $j$ in $\mathbf{Z}^{d}$. So it is easy to see that $\Theta^{2}(Q)=Q$ for any $Q \in \mathscr{A}$. The even part (resp. odd part) of $\mathscr{A}$ will be denoted by $\mathscr{A}_{+}$(resp. $\mathscr{A}_{-}$),

$$
\mathscr{A}_{+}=\{Q \in \mathscr{A} \mid \Theta(Q)=Q\}, \quad \mathscr{A}_{-}=\{Q \in \mathscr{A} \mid \Theta(Q)=-Q\} .
$$


The (global) gauge action $\gamma_{\theta}$ of $U(1)$ is defined by

$$
\gamma_{\theta}\left(a_{k}^{*}\right)=e^{i \theta} a_{k}^{*}, \quad \gamma_{\theta}\left(a_{k}\right)=e^{-i \theta} a_{k}
$$

for all $k$ in $\mathbf{Z}^{d}$. We denote the gauge invariant part of $\mathscr{A}$ by $\mathscr{A}^{U(1)}$,

$$
\mathscr{A}^{U(1)}=\left\{Q \in \mathscr{A} \mid \gamma_{\theta}(Q)=Q(\forall \theta)\right\} \text {. }
$$

By $\mathscr{B}$ we denote the abelian $C^{*}$-subalgebra of $\mathscr{A}^{U(1)}$ generated by all $n_{j}\left(j \in \mathbf{Z}^{d}\right)$. $\mathscr{B}$ is isomorphic to the set of continuous functions on $\{1,0\}^{\mathbf{Z}^{d}}$ as a $C^{*}$-algebra.

We now state our result on the existence of time evolution. We consider the following translationally invariant Hamiltonian $H$,

$$
H=\sum_{j \in \mathbf{Z}^{d}} \tau_{j}(h),
$$

where $h$ is a selfadjoint element of $\mathscr{A}_{+}$expanded by $b(A)$ of $(1.6)$ as follows:

$$
h=\sum_{A: A \ni 0} h(A) b(A) .
$$

$h(A)$ is a complex number and the above sum is taken for finite even subsets $\mathrm{A}$ of $\Gamma=\mathbf{Z}^{d} \times\{+,-\}$ which contain $(0,+)$ or $(0,-)$. The selfadjointness of $h$ is equivalent to the following identity:

$$
\bar{h}(A)=(-1)^{|A| / 2} h(A) .
$$

Theorem 1.1. Assume that the following is finite,

$$
\sum_{A: A \ni 0}|A|^{2}|h(A)|<\infty \text {. }
$$

Then the derivation $\delta$ defined by

$$
\delta(Q)=i[H, Q]=i \sum_{j \in \mathbf{Z}^{d}}\left[\tau_{j}(h), Q\right]
$$

is well defined on $\mathscr{A}_{\mathrm{loc}}$ and its closure generates the one-parameter group of automorphisms $\alpha_{t}(t \in R)$ of $\mathscr{A}$,

$$
\frac{d}{d t} \alpha_{t}(Q)=i \alpha_{t}([H, Q])
$$

for $Q$ in $\mathscr{A}_{\text {loc }}$.

The condition (1.10) is different from the conventional description for Hamiltonians. The statement of Theorem 1.1 is also valid for the following translationally invariant Hamiltonian (See Theorem 2.9.):

$$
h=\sum_{X \ni 0} \Psi(X)
$$

where $X$ is a finite subset of $\mathbf{Z}^{d}$ and $\Psi(X)=\Psi(X)^{*} \in \mathscr{A}_{+} \cap \mathscr{A}_{X}$,

$$
\sum_{X: X \ni 0}|X|^{2}|\Psi(X)|<\infty \text {. }
$$

Next we present a characterization for infinite volume ground states for the class of interactions with (1.10). Recall that the ground state is a state $\varphi$ of $\mathscr{A}$ 
satisfying

$$
\frac{1}{i} \varphi\left(Q^{*} \delta(Q)\right)=\varphi\left(Q^{*}[H, Q]\right) \geqq 0
$$

for any element $Q$ in the domain (or core) of the generator of our time evolution $\alpha_{t}$ of Theorem 1.1. A brief explanation for this definition will be given later in Sect. 3 . (See also Chapter 5 and 6 of [5].) The infinite volume ground state is characterized by minimization of the energy per volume in the sense specified below.

Theorem 1.2. Consider the translationally invariant Hamiltonian $H$ satisfying the condition of Theorem 1.1. Let $\varphi$ be a translationally invariant state of $\mathscr{A}$. Then the following conditions are equivalent:

1. $\varphi$ is a ground state.

2. $\varphi$ minimizes the energy per volume in the sense that

$$
\varphi(h)=\inf \psi(h),
$$

where inf is taken for all the translationally invariant states $\psi$

The above theorem was proved for quantum spin models (for example, Heisenberg models) by O. Bratteli, A. Kishimoto and Robinson in [4]. Our Theorem 1.2 is the Fermion version of their results.

Even though the Hamiltonian is translationally invariant, the ground state can be non-translationally invariant. In [10] C.T. Gottstein and R. Werner have shown that the 1 dimensional ferromagnetic anisotropic Heisenberg model has infinite volume non-translationally invariant ground states. Via the Jordan Wigner transform we obtain a Fermion Hamiltonian with infinite volume non-translationally invariant ground states. The explicit form of the Hamiltonian is

$$
H=-\sum_{j}\left\{a_{j+1}^{*} a_{j}+a_{j}^{*} a_{j+1}+\frac{\Delta}{2}\left(2 n_{j}-1\right)\left(2 n_{j+1}-1\right)\right\},
$$

where $\Delta$ is a real parameter satisfying $\Delta>1$. In Sect. 3 , we will also give a characterization of ground states which are not necessarily translationally invariant. (See Theorem 3.4.)

In physics, not only ground states for $\mathscr{A}$ but ground states in a fixed density of particles are considered. Consider the finite volume Hamiltonian $H_{\Lambda}$ on $\Lambda$ determined via the equation,

$$
H_{\Lambda}=\sum_{\Lambda \ni k, l} t_{k l} a_{k}^{*} a_{l}+\sum_{\Lambda \supset A} W_{A} \prod_{A \ni j} n_{j}
$$

Set

$$
n_{\Lambda}=\frac{\sum_{\Lambda \ni j} n_{j}}{|\Lambda|}
$$

Fix $s$ with $0<s<1$. For each finite volume $\Lambda$, consider $s_{\Lambda}\left(0<s_{\Lambda}<1\right)$ and a unit vector $\xi_{\Lambda}$ in the Fock space satisfying

$$
n_{\Lambda} \xi_{\Lambda}=s_{\Lambda}|\Lambda| \xi_{\Lambda}
$$

and

$$
\left(\xi_{\Lambda}, H_{\Lambda} \xi_{\Lambda}\right)=\inf \left(\omega, H_{\Lambda} \omega\right),
$$


where inf is taken among all the unit vectors $\omega$ in the Fock space with the same density condition,

$$
n_{\Lambda} \omega=s_{\Lambda}|\Lambda| \omega .
$$

$\xi_{\Lambda}$ is the ground state vector when the total number of particles is fixed. A typical question of the above Fermion model is the behaviour of the ground state vectors $\xi_{\Lambda}$ in the infinite volume limit under the condition, $\lim s_{\Lambda}=s$. So let $\psi$ be the infinite volume limit state of the vector states associated with $\xi_{\Lambda}$,

$$
\lim _{\Lambda \rightarrow \mathbf{Z}^{d}}\left(\xi_{\Lambda}, Q \xi_{\Lambda}\right)=\psi(Q) .
$$

It is easy to see that $\psi$ satisfies the ground state condition (1.13) for $Q$ in $\mathscr{A}^{U(1)} \cap$ $\mathscr{A}_{\text {loc }}$. We propose here our definition of the (infinite volume) states with particle density $s$, which substitutes for (1.16) when we handle the infinite volume states.

Definition 1.3. Let $P_{\Lambda}(s, \delta)$ be the projection to the eigenvectors of $n_{\Lambda}$ with the eigenvalue in $(s-\delta, s+\delta)$. A state $\psi$ of $\mathscr{A}^{U(1)}$ has the density $s$ iff $\psi$ is translationally invariant and for any positive $\delta$,

$$
\lim _{\Lambda \rightarrow \mathbf{Z}^{d}} \psi\left(P_{\Lambda}(s, \delta)\right)=1 .
$$

Any ergodic ground state of $\mathscr{A}^{U(1)}$ satisfying $\psi\left(n_{j}\right)=s$ is a state with density $s$ in our sense. (See Proposition 4.3.) By ergodic state we mean an extremal state in the set of translationally invariant states.

Theorem 1.4. Suppose that $h$ of (1.9) is gauge invariant, $h \in \mathscr{A}^{U(1)}$ and that (1.10) is valid.

1. Suppose that $\varphi$ is a translationally invariant state with density $s$. If it is a ground state for $h$ and $\mathscr{A}^{U(1)}$, we have

$$
\varphi(h)=\inf \psi(h),
$$

where inf is taken among states $\psi$ of $\mathscr{A}^{U(1)}$ with density $s=\varphi\left(n_{j}\right)$ in the sense of Definition 1.3.

2. Conversely, let $\varphi$ be a state of $\mathscr{A}^{U(1)}$ with density $s$ satisfying the condition (1.20). Then $\varphi$ is a ground state for $\mathscr{A}^{U(1)}$.

As a corollary to this theorem we also have

Theorem 1.5. Assume the condition of Theorem 1.4. Let $\varphi$ be an ergodic ground state for $h$ and $\mathscr{A}^{U(1)}$ with density s. Consider the gauge invariant extension $\tilde{\varphi}$, of $\varphi$ to $\mathscr{A}$,

$$
\tilde{\varphi} \circ \gamma_{\theta}=\tilde{\varphi}, \quad \varphi(Q)=\tilde{\varphi}(Q), \quad Q \in \mathscr{A}^{U(1)} .
$$

There exists $\mu$ such that $\tilde{\varphi}$ is a ground state for $\mathscr{A}$ with the Hamiltonian

$$
H(\mu)=\sum_{j} \tau_{j}(h)+\mu \sum_{j} n_{j} .
$$

The rest of this paper is organized as follows. In Sect. 2, we prove Theorem 1.1 and related results. Section 3 is devoted to Theorem 1.2. In Sect. 4 we discuss the characterization of ground states for $\mathscr{A}^{U(1)}$. 


\section{Time Evolution}

The aim of this chapter is to prove Theorem 1.1 and related results which we will use later.

Let us begin with the algebraic structure of the CAR alegbra $\mathscr{A}$. As a $C^{*}$ algebra, $\mathscr{A}$ is simple and it is isomorphic to the infinite tensor product of the 2 by 2 complex matrix algebra $M_{2}(C)$. In fact $\mathscr{A}_{\Lambda}$ is the algebra of $2^{|\Lambda|}$ by $2^{|\Lambda|}$ complex matrices $M_{2|\Lambda|}(C)$ which is isomorphic to the tensor product of the $|\Lambda|$ copies of $M_{2}(C)$. For later use, we give here the explicit form of isomorphism. As before the elements of $\Lambda$ are numbered, $\Lambda=\left\{j_{1} j_{2} \cdots j_{|\Lambda|}\right\}$, and set

$$
\begin{aligned}
& \sigma_{z}^{(l)}=2 n_{j_{l}}-1=2 a_{j_{l}}^{*} a_{j_{l}}-1, \\
& \sigma_{x}^{(l)}=\left(\prod_{k=1}^{l-1} 2 n_{j_{k}}-1\right) b_{(l,+)}, \\
& \sigma_{y}^{(l)}=\left(\prod_{k=1}^{l-1} 2 n_{j_{k}}-1\right) b_{(l,-)} .
\end{aligned}
$$

Then $\sigma_{\alpha}^{(k)}$ satisfies the commutation relation of Pauli matrices,

$$
\begin{aligned}
\sigma_{\alpha}^{(l)} & =\sigma_{\alpha}^{(l)^{*}}, \quad \sigma_{\alpha}^{(l)^{2}}=1, \\
\sigma_{\alpha}^{(l)} \sigma_{\beta}^{(k)} & =\sigma_{\beta}^{(k)} \sigma_{\alpha}^{(l)} \quad(k \neq l), \\
\sigma_{\alpha}^{(l)} \sigma_{\beta}^{(l)} & =-\sigma_{\beta}^{(l)} \sigma_{\alpha}^{(l)} \quad(\alpha \neq \beta) .
\end{aligned}
$$

$\sigma_{\alpha}^{(k)}$ generates $M_{2|\Lambda|}(C)$. It is also known that $\mathscr{A}_{+}$is isomorphic to $\mathscr{A}$ as a $C^{*}$ algebra. Then the following lemma is obvious once we realize the above isomorphism of the Pauli spin algebra and the CAR algebra.

Lemma 2.1. Let $\Lambda^{\prime}$ be a (not necessarily finite) subset of $\mathbf{Z}^{d}$ which includes a finite subset $\Lambda, \Lambda^{\prime} \supset \Lambda$. Let $Q$ be an operator in $\mathscr{A}_{\Lambda^{\prime}}$ which commutes with $\mathscr{A}_{\Lambda}, Q R=R Q$ for any $R \in \mathscr{A}_{\Lambda}$. Then $Q$ is written in the following way:

$$
Q=Q_{+}+Q_{-} V_{\Lambda}, \quad Q_{ \pm} \in \mathscr{A}_{\Lambda^{\prime} \cap A^{c}} \cap \mathscr{A}_{ \pm},
$$

where $\Lambda^{c}$ is the complement of $\Lambda$ and $V_{\Lambda}$ is defined by

$$
V_{\Lambda}=\prod_{j \in \Lambda}\left(2 n_{j}-1\right) .
$$

By $\mathscr{A}_{\Lambda}^{c}$ we will denote the commutant of $\mathscr{A}_{\Lambda}, \mathscr{A}_{\Lambda}^{c}=\left\{Q \mid Q R=R Q\left(\forall R \in \mathscr{A}_{\Lambda}\right)\right\}$. Next we introduce the variation norm following [13]. For $\alpha$ in $\Gamma, \partial_{\alpha}$ is a linear operator on $\mathscr{A}$ determined via the equation,

$$
\partial_{\alpha}(Q)=1 / 2\left(b_{\alpha} \Theta(Q) b_{\alpha}-Q\right) .
$$

Then it is easy to see the following relations:

$$
\begin{gathered}
\partial_{\alpha}{ }^{2}=-\partial_{\alpha}, \quad \partial_{\alpha} \circ \partial_{\beta}=\partial_{\beta} \circ \partial_{\alpha}, \\
\partial_{\alpha}\left(b_{\beta}\right)=\left\{\begin{array}{ll}
0, & \text { if } \beta \neq \alpha \\
-b_{\alpha}, & \text { if } \beta=\alpha
\end{array} .\right.
\end{gathered}
$$


We now define $\|||||$ and \|\|$_{C^{1}}$ via the following equations:

$$
\||Q|\|=\sum_{\alpha \in \Gamma}\left\|\partial_{\alpha}(Q)\right\|, \quad\|Q\|_{C^{1}}=\|Q\|+\|\mid Q\| \|
$$

where $\|Q\|$ is the $C^{*}$-algebra norm of $Q$ in $\mathscr{A}$. By $C^{1}(\mathscr{A})$ we denote the set of all $\mathrm{Q}$ with finite $\||Q|\|$,

$$
C^{1}(\mathscr{A})=\{Q \in \mathscr{A}|\||Q|\|<\infty\} .
$$

Historically, $C^{1}(\mathscr{A})$ and $\|||||$ were introduced for classical Ising spin systems (cf. [11]) and we defined the same objects for the quantum spin case in [13]. S. Richter and $\mathrm{R}$. Werner have investigated various properties of the (more general) variational norm for non-commutative $C^{*}$-algebras in [16]. We present here some facts on $C^{1}(\mathscr{A})$ and $\||| \mid$.

Lemma 2.2. (i) $\||Q|\|=0$ iff $Q$ is a scalar, $Q=c 1$.

(ii) If $Q$ is in $C^{1}(\mathscr{A})$,

$$
\|Q-\operatorname{tr}(Q) 1\| \leqq 3 / 2\|\mid Q\| \|
$$

where $\operatorname{tr}$ is the unique normalized trace of $\mathscr{A}$.

(iii) $C^{1}(\mathscr{A})$ is a Banach algebra with the norm \|\|$_{C^{1}}$.

(iv) $\mathscr{A}_{\text {loc }}$ is dense in $C^{1}(\mathscr{A})$ with respect to the norm \|\|$_{C^{1}}$.

Proof of Lemma 2.2. (i) and (iii) are obvious. (iv) is proved in Proposition 9 of [16]. To show (ii), consider the partial trace $\operatorname{tr}_{\Lambda}$ on $\mathscr{A}_{\Lambda}$ determined by

$$
\operatorname{tr}_{\Lambda}\left(Q_{1} Q Q_{2}\right)=Q_{1} \operatorname{tr}(Q) Q_{2} \quad\left(Q \in \mathscr{A}_{\Lambda} \quad Q_{1}, Q_{2} \in \mathscr{A}_{\Lambda^{c}}\right) .
$$

For the one point algebra $\mathscr{A}_{\{j\}}$ the partial trace is obtained via the following formula,

$$
\operatorname{tr}_{\{j\}}(Q)=Q+\partial_{(j,+)}(Q)+\partial_{(j,-)}(Q)+\partial_{(j,+)} \circ \partial_{(j,-)}(Q) .
$$

The above trace formula follows from (2.6) and (2.7). On the other hand, we have

$$
\operatorname{tr}_{\Lambda \cup\{j\}}(Q)=\operatorname{tr}_{\Lambda}\left(\operatorname{tr}_{\{J\}}(Q)\right)
$$

and

$$
\left\|\partial_{(j,+)} \circ \partial_{(j,-)}(Q)\right\| \leqq\left\|\partial_{(j, \pm)}(Q)\right\|
$$

The latter inequality implies

$$
\left\|\partial_{(j,+)} \circ \partial_{(j,-)}(Q)\right\| \leqq 1 / 2\left\|\partial_{(j,+)}(Q)\right\|+1 / 2\left\|\partial_{(j,-)}(Q)\right\| .
$$

Then

$$
\begin{gathered}
Q-\operatorname{tr}(Q) 1=\left(\operatorname{tr}_{\{j\}}(Q)-\operatorname{tr}(Q) 1\right)-\partial_{(j,+)}(Q)-\partial_{(j,-)}(Q)-\partial_{(j,+)} \circ \partial_{(j,-)}(Q), \\
\|Q-\operatorname{tr}(Q)\| \leqq\left\|\operatorname{tr}_{\{j\}}(Q)-\operatorname{tr}(Q)\right\|+3 / 2 \sum_{a=(j,+),(j,-)}\left\|\partial_{a}(Q)\right\|
\end{gathered}
$$


By iterating this procedure, we have

$$
\|Q-\operatorname{tr}(Q) 1\| \leqq\left\|\operatorname{tr}_{\Lambda}(Q)-\operatorname{tr}(Q) 1\right\|+3 / 2 \sum_{a=(j, \pm), j \in \Lambda}\left\|\partial_{a}(Q)\right\| .
$$

This implies (ii).

Next we turn to the generator of the time evolution.

Lemma 2.3. Assume that the Hamiltonian $H$ of (1.8) (or $h$ of (1.9)) satisfies condition (1.10). Consider the derivation $\delta$ determined by (1.11).

$\delta$ is well-defined on $C^{1}(\mathscr{A})$. There exists a positive constant $K$ such that

$$
\|\delta(Q)\|=\|[H, Q]\| \leqq K\||Q|\|
$$

for any $Q$ in $C^{1}(\mathscr{A})$.

Proof of Lemma 2.3. For $b_{\alpha}$ defined in (1.4), and for $Q$ in $\mathscr{A}_{ \pm}$, the following can be checked by hand:

$$
\left\|\left[b_{\alpha} b_{\beta}, Q\right]\right\| \leqq 2\left(\left\|\partial_{\alpha}(Q)\right\|+\left\|\partial_{\beta}(Q)\right\|\right) .
$$

Then set $Q_{ \pm}=1 / 2(Q \pm \Theta(Q))$. It turns out

$$
\left\|\partial_{\alpha}\left(Q_{ \pm}\right)\right\| \leqq\left\|\partial_{\alpha}(Q)\right\|
$$

and

$$
\begin{aligned}
\left\|\left[b_{\alpha} b_{\beta}, Q\right]\right\| & \leqq\left\|\left[b_{\alpha} b_{\beta}, Q_{+}\right]\right\|+\left\|\left[b_{\alpha} b_{\beta}, Q_{-}\right]\right\| \\
& \leqq 2 \sum_{c= \pm}\left(\left\|\partial_{\alpha}\left(Q_{c}\right)\right\|+\left\|\partial_{\beta}\left(Q_{c}\right)\right\|\right) \leqq 4\left(\left\|\partial_{\alpha}(Q)\right\|+\left\|\partial_{\beta}(Q)\right\|\right)
\end{aligned}
$$

Thus for $A=\left\{\alpha_{1} \alpha_{2}, \ldots, \alpha_{2 k}\right\} \subset \Gamma$, we have

As a result,

$$
\|[b(A), Q]\| \leqq 4 \sum_{l=1,2,, k}\left\|\left[b_{\alpha-2 l-1} b_{\alpha_{2 l}}, Q\right]\right\| \leqq 4 \sum_{l=1,2,, 2 k}\left\|\partial_{\alpha_{l}}(Q)\right\| .
$$

$$
\|[H, Q]\|=\sum_{j \in \mathbf{Z}^{d}} \sum_{A}|h(A)|\|[b(A+j), Q]\| \leqq 4 \sum_{j \in \mathbf{Z}^{d}} \sum_{A}|h(A-j)| \sum_{\alpha \in A}\left\|\partial_{\alpha}(Q)\right\| .
$$

In this sum, for fixed $A$ (or $|h(A)|$ ) and $\alpha$, we have at most the cardinality $|A|$ of $A$ for $j$ 's so (2.15) is bounded from above by $4 \sum_{A}|h(A)\|A|\||Q|\|$.

Lemma 2.4. We assume that (1.10) is valid. For $Q=Q^{*}$ in $C^{1}(\mathscr{A})$ and a positive real number $\lambda$, consider $R$ determined by

$$
R=Q-i \lambda[H, Q]=(1-\lambda \delta)(Q) .
$$

Let $D=\left\{D_{a b}\right\}$ be a positive infinite matrix acting on $l^{1}(\Gamma)$ defined by

$$
\left.\sum_{b \in \Gamma} D_{a b} f_{b}=2 \lambda \sum_{j \in \Gamma} \sum_{A: A+j \ni a}|h(A)|\left(\sum_{b: A+j \ni b} f_{b}\right) \quad \text { for } f=\left\{f_{b} \mid b \in \Gamma\right\} \in l^{1}(\Gamma)\right\} .
$$

Then

$$
\left\|\partial_{\alpha}(Q)\right\|-\sum_{\beta} \lambda D_{\alpha \beta}\left\|\partial_{\beta}(Q)\right\| \leqq\left\|\partial_{\alpha}(R)\right\|
$$


Proof of Lemma 24 For a selfadjoint $Q$, the spectrum of $\partial_{\alpha}(Q)$ is symmetric with respect to 0 because $b_{\alpha}$ is a selfadjoint unitary and

$$
b_{\alpha} \Theta\left(\partial_{\alpha}(Q)\right) b_{\alpha}=-\partial_{\alpha}(Q) \text {. }
$$

So there exists a state $\varphi$ satisfying $\varphi\left(\partial_{\alpha}(Q)\right)=\left\|\partial_{\alpha}(Q)\right\|$. For such a state $\varphi$, we have

$$
\varphi\left(\partial_{\alpha}(Q)\right)=\left\|\partial_{\alpha}(Q)\right\| \geqq \varphi\left(e^{i t O} \partial_{\alpha}(Q) e^{-i t O}\right)
$$

for any selfadjoint $Q$. By differentiating this, we obtain

$$
\varphi\left(\left[O, \partial_{\alpha}(Q)\right]\right)=0 .
$$

We now return to the definition of $R$. Then,

$$
\begin{gathered}
\partial_{\alpha}(Q)=\partial_{\alpha}(R)+i \lambda \partial_{\alpha}([H, Q]), \\
\partial_{\alpha}(i[H, Q])=i \sum_{j \in \mathbf{Z}^{d}} \sum_{A} h(A)\left\{\left[\partial_{\alpha}(b(A+j)), b_{\alpha} \Theta(Q) b_{\alpha}\right]+\left[b(A+j), \partial_{\alpha}(Q)\right]\right\} .
\end{gathered}
$$

By (2.18) we have

$$
\begin{aligned}
\left\|\partial_{\alpha}(Q)\right\| & =\varphi\left(\partial_{\alpha}(Q)\right) \\
& =\varphi\left(\partial_{\alpha}(R)\right)+i \lambda \sum_{j \in \mathbf{Z}^{d}} \sum_{A} h(A) \varphi\left(\left[\partial_{\alpha}(b(A+j)), b_{\alpha} \Theta(Q) b_{\alpha}\right]\right) \\
& \leqq\left\|\partial_{\alpha}(R)\right\|+\lambda \sum_{j \in \mathbf{Z}^{d}} \sum_{A} \mid h(A)\|\|\left(\left[\partial_{\alpha}(b(A+j)), Q\right]\right) \| \\
& \leqq\left\|\partial_{\alpha}(R)\right\|+\lambda \sum_{j \in \mathbf{Z}^{d}} \sum_{A: A+j \ni a} \mid h(A)\|\|([b(A+j), Q]) \| \\
& \leqq\left\|\partial_{\alpha}(R)\right\|+2 \lambda \sum_{j \in \mathbf{Z}^{d}} \sum_{A: A+j \ni a}|h(A)| \sum_{\alpha: A+j \ni \alpha}\left\|\partial_{\alpha}(Q)\right\| \\
& =\left\|\partial_{\alpha}(R)\right\|+\sum_{\beta} \lambda D_{\alpha \beta}\left\|\partial_{\beta}(Q)\right\| .
\end{aligned}
$$

Lemma 2.5. Under the assumption (1.10), the linear operator D of Lemma 2 is a bounded operator on $l^{1}(\Gamma)$ and its operator norm is estimated as follows:

$$
\|D\|_{l^{1}(\Gamma)} \leqq M=2 \lambda \sum_{A}|h(A) \| A|^{2}
$$

Proof of Lemma 2.5 We begin with

$$
\sum_{a} D_{a b}=2 \lambda \sum_{j \in \Gamma} \sum_{a} \sum_{A: A+j \ni a} \sum_{b: A+j \ni b}|h(A)| .
$$

This summation reads as follows. For a fixed $A$ or $h(A)$, consider a pair $(x, y)$ of points in $A$ such that $x=a-j, y=b-j$. As $b$ is fixed, $j$ satisfying $y=b-j$ is unique so that $a$ is determined automatically. Thus the possibile choice for $a, b, j$ is at most that for $(x, y)$ in $A \times A$ and $|h(A)|$ appears at most $|A| \times|A|$. As a result we have

$$
\sum_{a} D_{a b} \leqq 2 \lambda \sum_{A}|h(A)||A|^{2}
$$


Now we can estimate the norm of $D$. For $f=\left\{f_{a}\right\} \in l^{1}(\Gamma)$,

$$
\begin{aligned}
\|D f\|_{l^{1}(\Gamma)} & =\sum_{a, b} D_{a b}\left|f_{b}\right| \leqq \sum_{b}\left(\sum_{a} D_{a b}\right)\left|f_{b}\right| \\
& \leqq M \sum_{a}\left|f_{a}\right|=M\|f\|_{l^{1}(\Gamma)} \cdot \square
\end{aligned}
$$

Proof of Theorem 1.1. For $\delta(Q)=i[H, Q]$ and $\lambda$ satisfying $\left|\frac{\lambda}{M}\right|<1$ we show that $(1-\lambda \delta)\left(C^{1}(\mathscr{A})\right)$ contains $C^{1}(\mathscr{A})$. This is sufficient for our purpose as this implies that $1-\lambda \delta$ has the dense range and the range of its closure is total so we can use general results such as Lemma 3.1.14, Lemma 3.1.15 and Theorem 3.1.10 of [5].

To prove $(1-\lambda \delta)\left(C^{1}(\mathscr{A})\right)$ contains $C^{1}(\mathscr{A})$ we may and do restrict our attention to the selfadjoint part. So take $R=R^{*} \in C^{1}(\mathscr{A})$. Consider the bounded derivation $\delta^{(n)}$ determined by

$$
\delta^{(n)}(Q)=i \sum_{j \in \mathbf{Z}^{d}:|j|<n}\left[\tau_{j}(h), Q\right] .
$$

As $\delta^{(n)}$ is a bounded generator we can find $Q_{n}$ determined by

$$
Q_{n}-\lambda \delta^{(n)}\left(Q_{n}\right)=R \text {. }
$$

We claim that $Q_{n} \in C^{1}(\mathscr{A})$. To show this we may assume that $\lambda$ is positive. Then the proof of Lemma 2.4 implies

$$
\left\|\partial_{a}\left(Q_{n}\right)\right\|-\lambda \sum_{b} D_{a b}\left\|\partial_{b}\left(Q_{n}\right)\right\| \leqq\left\|\partial_{a}(R)\right\| .
$$

$D=\left\{D_{a b}\right\}$ is a positive matrix, and $(1-\lambda D)^{-1}$ is also a positive matrix due to the convergent Neumann expansion, $(1-\lambda D)^{-1}=\sum_{k=0,1,2} D^{k}$. As a consequence, Eq. (2.24) leads us to

$$
\left.\left\|\partial_{a}\left(Q_{n}\right)\right\| \leqq \sum_{b}(1-\lambda D)^{-1}\right)_{a b}\left\|\partial_{b}(R)\right\| .
$$

By the assumption $\left|\frac{\lambda}{M}\right|<1$ we have the following:

$$
\left\|\left|Q_{n}\right|\right\| \leqq \sum_{k}\left|\frac{\lambda}{M}\right|^{k}\||R|\| .
$$

Now that $Q_{n} \in C^{1}(\mathscr{A})$, we can define $R_{n}$ via the equation

$$
Q_{n}-\lambda \delta\left(Q_{n}\right)=R_{n} .
$$

Then

$$
\begin{aligned}
\left\|R-R_{n}\right\| & \leqq \lambda \sum_{j \in \mathbf{Z}^{d}:|j| \geqq n}\left\|\left[\tau_{j}(h), Q_{n}\right]\right\| \\
& \leqq 2 \lambda \sum_{|j| \geqq n} \sum_{A}|h(A)| \sum_{a: A+j \in a}\left\|\partial_{a}\left(Q_{n}\right)\right\| \\
& \left.\leqq 2 \lambda \sum_{|j| \geqq n} \sum_{A}|h(A)| \sum_{a: A+j \in a} \sum_{b}(1-\lambda D)^{-1}\right)_{a b}\left\|\partial_{b}(R)\right\|
\end{aligned}
$$


The right-hand side of (2.26) is finite even for $n=0$. In fact

$$
\begin{aligned}
& \left.2 \lambda \sum_{j} \sum_{A}|h(A)| \sum_{a: A+j \in a} \sum_{b}(1-\lambda D)^{-1}\right)_{a b}\left\|\partial_{b}(R)\right\| \\
& \left.\quad \leqq 2 \lambda \sum_{A}|h(A)||A| \sum_{a \in \Gamma} \sum_{b}(1-\lambda D)^{-1}\right)_{a b}\left\|\partial_{b}(R)\right\| \\
& \quad \leqq 2 \lambda \sum_{A}\left|h(A)\|A \mid\|(1-\lambda D)^{-1}\right) f \|_{l^{1}(\Gamma)}<\infty
\end{aligned}
$$

for $f_{a}=\left\|\partial_{a}(R)\right\|$. Thus in (2.26) if we take $n$ to the infinite,

$$
\lim _{n \rightarrow \infty}\left\|R-R_{n}\right\|=0
$$

which completes the proof of Theorem 1.1.

For later use, we introduce a class of Feller semigroups. (See also [14].) First we explain what a Feller semigroup on a $C^{*}$-algebra means. Let $\mathscr{C}$ be a $C^{*}$-algebra. A linear map $E$ on $\mathscr{C}$ is positive if $E$ preserves the positivity of spectrum, $E\left(Q^{*} Q\right) \geqq 0$ for any $Q \in \mathscr{C}$. Given a linear map $E$ on $\mathscr{C}$, we define a linear map $E_{n}$ on $\mathscr{C} \otimes M_{n}(C)=M_{n}(\mathscr{C})$ by the equation, $E_{n}=\Psi \otimes 1$ or equivalently, $E_{n}\left(\left[a_{i j}\right]\right)=\left[E\left(a_{i j}\right)\right]$, where $\mathscr{C} \otimes M_{n}(C)$ is identified with $\mathscr{C}$ valued $n$ by $n$ matrices $M_{n}(\mathscr{C})$.

Definition 2.6. A linear map $E$ on a $C^{*}$-algebra is completely positive if $E_{n}$ is positive for any natural number $n$.

For example, let $d$ be an element of $\mathscr{C}$ and set $E(Q)=d^{*} Q d$. $E$ is completely positive. More generally the map determined by the following equation is completely positive if it converges:

$$
E(Q)=\sum_{j} d_{j}^{*} Q d_{j}
$$

where $d_{j}$ are elements of $\mathscr{C}$.

Completely positive maps are basic ingredients of the quantum theory of open systems. (See [6].) Fundamental mathematical facts on completely positive maps may be found in [15].

Definition 2.7. Let $\mathscr{C}$ be a unital $C^{*}$-algebra. A strongly continuous semigroup of unit preserving completely positive maps on $\mathscr{C}$ is called a Feller semigroup.

If $\mathscr{C}$ is the matrix algebra $M_{n}(C)$, the generator $L$ of a Feller semigroup $S_{t}$ is always written in the following form:

$$
L(Q)=E(Q)-1 / 2\{E(1), Q\}+i[H, Q]
$$

where $E$ is a completely positive map on $\mathscr{B}, H=H^{*}$ is a selfadjoint element of $\mathscr{C}$. As this form was first discovered by G. Lindblad, it is referred to as the Lindblad generator (cf. [12]). 
Now let us consider the translationally invariant (pre)generators of Feller semigroups on $\mathscr{A}$ determined by

$$
\begin{gathered}
L(Q)=\sum_{j \in \mathbf{Z}^{d}} L_{j}(Q), \\
L_{j}(Q)=\tau_{j} \circ L_{0} \circ \tau_{-j}(Q), \\
L_{0}(Q)=E(Q)-1 / 2\{E(1), Q\}+i[h, Q],
\end{gathered}
$$

where $E$ is a completely positive map and $h$ is a selfadjoint element of $\mathscr{A}$. We assume the following condition for the completely positive map $E$ in (2.29):

$$
\begin{gathered}
E=E^{+}+E^{-}, \\
E^{+}(Q)=\sum_{k=1}^{\infty} d(k,+)^{*} Q d(k,+), \\
E^{-}(Q)=\sum_{k=1}^{\infty} d(k,-)^{*} \Theta(Q) d(k,-),
\end{gathered}
$$

where $d(k,+)$ is an even element and $d(k,-)$ is an odd element, $d(k,+) \in \mathscr{A}_{+}$, $d(k,-) \in \mathscr{A}_{-}$. The odd part $E_{-}$of $E$ is studied systematically in [7].

Suppose that $d(k+), d(k-)$ and $h$ are written in the following way:

$$
\begin{aligned}
d(k+) & =\sum_{A} D_{k}(A,+) b(A), \\
d(k-) & =\sum_{A} D_{k}(A,-) b(A), \\
h & =\sum_{A} h(A) b(A),
\end{aligned}
$$

where $D_{k}(A,+), D_{k}(A,-)$ and $h(A)$ are constants and the sums of (2.32) and (2.34) are taken for even finite subsets of $\Gamma$ ( $A$ with an even number of elements) while the sum of (2.33) is taken for odd finite subsets.

Theorem 2.8. Consider $L$ determined by (2.29)-(2.34). Assume that $h$ of (2.34) is selfadjoint and that the following sums are finite:

$$
\sum_{k} \sum_{A}|D(A, \pm)||A|^{2}<\infty, \quad \sum_{A}|h(A)||A|^{2}<\infty
$$

Then, $L$ is well-defined on $\mathscr{A}_{\mathrm{loc}}$ and its closure $\bar{L}$ generates the Feller semigroup $S_{t}$ on $\mathscr{A}, \frac{d}{d t} S_{t}(Q)=S_{t}(L(Q))$. Furthermore, there exists a positive constant $K$ such that

$$
\left\|\left|S_{t}(Q)\right|\right\| \leqq 2 e^{K t}\||Q|\|
$$

for any $Q$ in $C^{1}(\mathscr{A})$. 
This is a generalization of Theorem 1.1 and can be proved in the same manner. The description of interactions of Hamiltonians in Theorems 1.1 and 2.8 are slightly different from the conventional way. Given $h$ or $d(k, \pm)$ the coefficient $h(A)$ and $D_{k}(A, \pm)$ are canonically determined. For example, set

$$
h(A)=\operatorname{tr}\left(b(A)^{-1} h\right) .
$$

Then (1.9) converges in the $L^{2}$ norm of the canonical trace of $\mathscr{A}$. A conventional way for presentation $h$ is as follows:

$$
h=\sum_{X \subset \mathbf{Z}^{d}} \Psi(X),
$$

where $\Psi(X) \in \mathscr{A}_{X}$. This presentation is far from unique. We can prove results corresponding to Theorems 1.1 and 2.8 in this presentation.

Theorem 2.9. Suppose that

$$
h=\sum_{X \subset \mathbf{Z}^{d}} \Psi(X)
$$

where $\Psi(X) \in \mathscr{A}_{+} \cap \mathscr{A}_{X}$ and

$$
\sum_{X \subset \mathbf{Z}^{d}}\|\Psi(X)\||X|^{2}<\infty
$$

Consider the derivation $\delta$ determined by (1.11). The closure of $\delta$ is the generator of a one-parameter group of automorphisms of $\mathscr{A}$.

The only differences in our proof of Theorems 1.1 and 2.9 are the estimates (2.15) and (2.19). If $A$ is in $\mathscr{A}_{+} \cap \mathscr{A}_{X}$ then

$$
[A, R]=\left[A, R-\operatorname{tr}_{X}(R)\right] .
$$

On the other hand, if $j$ is in $X$,

$$
Q-\operatorname{tr}_{X}(Q)=Q-\operatorname{tr}_{X-\{j\}}(Q)+\operatorname{tr}_{X-\{j\}}\left(Q-\operatorname{tr}_{j}(Q)\right),
$$

so

$$
\left\|Q-\operatorname{tr}_{X}(Q)\right\| \leqq \sum_{j \in X}\left\|Q-\operatorname{tr}_{j}(Q)\right\|
$$

Combined with (2.11) we have

$$
\begin{aligned}
\|[A, Q]\| & \leqq 2\|A\|\left\|Q-\operatorname{tr}_{X}(Q)\right\| \\
& \leqq 2\|A\| \sum_{j \in X}\left\|Q-\operatorname{tr}_{j}(Q)\right\| \\
& \leqq 2\|A\| \sum_{j \in X}\left(\left\|\partial_{j,+}(Q)\right\|+\left\|\partial_{j,-}(Q)\right\|\right) .
\end{aligned}
$$

With this estimate it is straightforward to prove Lemmas 2.4 and 2.5 in the context of Theorem 2.9. 


\section{Ground State}

Let us recall the definition of a ground state for general quantum systems. Let $\mathscr{C}$ be a $C^{*}$-algebra with the unit element. Let $\alpha_{t}$ be a strongly continuous one-parameter group of automorphisms of $\mathscr{C}$. (We regard $\alpha_{t}$ as the time evolution of the system.)

Definition 3.1. Let $\varphi$ be a state of $\mathscr{C}$. $\varphi$ is a ground state for $\alpha_{t}$ iff the following condition is valid for any $Q$ in the domain of the generator $\delta$ of $\alpha_{t}$ :

$$
-i \varphi\left(Q^{*} \delta(Q)\right) \geqq 0 .
$$

This definition makes sense for any $C^{*}$-algebra, in particular, for infinite quantum lattice systems. The physical meaning is as follows (cf. [5]).

Given a state $\varphi$, there exists a Hilbert space $\mathscr{H}_{\varphi}$, a unit vector $\Omega_{\varphi}$ in $\mathscr{H}_{\varphi}$, a representation $\pi_{\varphi}()$ of $\mathscr{A}$ on $\mathscr{H}_{\varphi}$ such that

$$
\varphi(Q)=\left(\Omega_{\varphi}, \pi_{\varphi}(Q) \Omega_{\varphi}\right) .
$$

This is the so-called GNS triple. If the state $\varphi$ is a ground state, there exists a positive selfadjoint operator $H \geqq 0$ on $\mathscr{H}_{\varphi}$ such that

$$
H \Omega_{\varphi}=0, \quad e^{\imath t H} \pi_{\varphi}(Q) e^{-i t H}=\pi_{\varphi}\left(\alpha_{t}(Q)\right)
$$

for any $Q$ in $\mathscr{A}$.

Conversely, suppose we have a representation $\pi$ of $\mathscr{A}$ on a Hilbert space $\mathscr{H}$, a positive selfadjoint operator $H \geqq 0$ satisfying $e^{i t H} \pi(Q) e^{-i t H}=\pi\left(\alpha_{t}(Q)\right)$. Suppose that the vector $\Omega$ is a unit eigenvector for $H$ with eigenvalue $0(H \Omega=0)$. The vector state $\varphi_{\Omega}$ associated with $\Omega,\left(\varphi_{\Omega}(Q)=(\pi(Q) \Omega, \Omega)\right)$ is a ground state. Thus the ground state in the sense of (3.1) is the vacuum expectation value. We can also show that any zero temperature limit of Gibbs states is a ground state in the sense of (3.1).

We present some facts which we use in this article. Proofs may be found in [5].

Proposition 3.2. (i) For a fixed time evolution $\alpha_{t}$, the set of all ground states is a convex weak*-closed subset of the state space

(ii) The set of all ground states is a face in the state space, namely, if a ground state $\varphi$ is a convex combination of other states $\varphi_{1}, \varphi, \varphi=\lambda \varphi_{1}+$ $(1-\lambda) \varphi_{2}$ with $0<\lambda<1, \varphi_{1}$ and $\varphi_{2}$ are also ground states

(iii) Any extremal ground state is pure (extremal in the set of all states). So if $\varphi$ is an extremal ground state and it is a convex sum of other states $\varphi_{1}$ and $\varphi_{2}$, $\varphi=\lambda \varphi_{1}+(1-\lambda) \varphi_{2}(0<\lambda<1)$, then $\varphi=\varphi_{1}=\varphi_{2}$

(iv) Let $\varphi$ be a ground state The positive selfadjoint operator $H$ satisfying (3.1) is affiliated with the von Neumann algebra generated by $\pi_{\varphi}(\mathscr{A})$, namely $e^{i t H} \in$ $\pi_{\varphi}(\mathscr{A})^{\prime \prime}$.

In what follows, we use a different presentation for our Hamiltonian.

Definition 3.3. An interaction is a collection of selfadjoint elements $\Psi(X)$ such that $X$ is a finite subset of $\mathbf{Z}^{d}, \Psi(X)=\Psi(X)^{*} \in \mathscr{A}_{+} \cap \mathscr{A}_{X}$ and

$$
\sum_{X: X \ni 0}\|\Psi(X)\|<\infty .
$$

An interaction is translationally invariant iff $\Psi(X+j)=\tau_{j}(\Psi(X))$ for any $j \in \mathbf{Z}^{d}$ and any $X \subset \mathbf{Z}^{d}$. 
Given an interaction and a finite subset $\Lambda$ of $\mathbf{Z}^{d}$, we set

$$
\begin{aligned}
& H_{\Lambda}=\sum_{X: \Lambda \supset X} \Psi(X), \\
& B_{\Lambda}=\sum_{X: \Lambda \cap X \neq \emptyset, \Lambda^{c} \cap X \neq \emptyset} \Psi(X) .
\end{aligned}
$$

Theorem 3.4. Suppose that a translationally invariant interaction $\left\{\Psi(A) \mid A \subset \mathbf{Z}^{d}\right\}$ is given and that the following derivation $\delta$ is well defined on $\mathscr{A}_{\text {loc }}$ :

$$
\delta(Q)=\lim _{\Lambda \rightarrow \mathbf{Z}^{d}}\left[H_{\Lambda}, Q\right],
$$

where the limit of $\Lambda$ is taken for $d$ dimensional cubes. We also assume that the closure of $\delta$ is the generator of the one-parmeter group of automorphisms $\alpha_{t}$ of $\mathscr{A}$ and

$$
\alpha_{t}(Q)=\lim _{\Lambda \rightarrow \mathbf{Z}^{d}} e^{\imath t H_{\Lambda}} Q e^{-i t H_{\Lambda}} .
$$

For a state $\varphi$ of $\mathscr{A}$, the following conditions are equivalent:

1. $\varphi$ is a ground state.

2. For any subset $\Lambda$ of $\mathbf{Z}^{d}$,

$$
\varphi\left(H_{\Lambda}+B_{\Lambda}\right)=\inf \psi\left(H_{\Lambda}+B_{\Lambda}\right),
$$

where inf is taken for all the states $\psi$ satisfying

$$
\psi(Q)=\varphi(Q)
$$

for any $Q$ in $\mathscr{A}_{\Lambda^{c}}$.

Proof of Theorem 3.4. The proof of this theorem is almost the same as that for Theorem 6 of [5]. We explain the difference originating from anti-commutativity.

First we assume that $\varphi$ is a $\Theta$ invariant ground state. We may also suppose that the state $\psi$ satisfying the condition (3.9) is $\Theta$ invariant due to the $\Theta$ invariance of $H_{\Lambda}$ and $B_{\Lambda}$. Let $\left\{\pi_{\varphi}(), \mathscr{H}_{\varphi}, \Omega_{\varphi}\right\}$ be the GNS triple for the $\Theta$ invariant ground state $\varphi$. Let $H$ be the positive selfadjoint operator on $\mathscr{H}_{\varphi}$ satisfying (3.2). By $\Theta$ invariance, we can define the unitary $U_{\Theta}$ which implements the automorphism $\Theta$,

$$
U_{\Theta} \pi_{\varphi}(Q) U_{\Theta}^{*}=\pi_{\varphi}(\Theta(Q)), \quad U_{\Theta} \Omega_{\varphi}=\Omega_{\varphi} .
$$

First we claim that $e^{i t H} \in \pi_{\varphi}\left(\mathscr{A}_{+}\right)^{\prime \prime}$. This follows from the fact that $\alpha_{t}$ commutes with $\Theta$ and

$$
U_{\Theta} e^{i t H} \pi_{\varphi}(Q) \Omega_{\varphi}=\pi_{\varphi}\left(\Theta\left(\alpha_{t}(Q)\right)\right) \Omega_{\varphi}=\pi_{\varphi}\left(\alpha_{t}(\Theta(Q))\right) \Omega_{\varphi}=e^{i t H} U_{\Theta} \pi_{\varphi}(Q) \Omega_{\varphi} .
$$

So

$$
U_{\Theta} e^{i t H}=e^{i t H} U_{\Theta} .
$$

By the Trotter-Kato formula,

$$
e^{i t\left(H-H_{A}-B_{\Lambda}\right)} \in \pi_{\varphi}\left(\mathscr{A}_{+}\right)^{\prime \prime} .
$$


On the other hand, for any $Q \in \mathscr{A}_{\Lambda}$,

$$
e^{i t\left(H-H_{\Lambda}-B_{\Lambda}\right)} Q=Q e^{i t\left(H-H_{\Lambda}-B_{\Lambda}\right)} .
$$

So

$$
e^{i t\left(H-H_{A}-B_{A}\right)} \in \pi_{\varphi}\left(\mathscr{A}_{+}\right)^{\prime \prime} \cap \pi_{\varphi}\left(\mathscr{A}_{\Lambda}\right)^{\prime} .
$$

$\psi$ satisfying the condition (3.9) is quasi-equivalent to $\varphi$ because of $\Theta$ invariance of $\varphi$ and $\psi$, Lemma 2.1 and Lemma 6.2 .55 of [5]. By $\bar{\psi}$ we denote the normal extension of $\psi$ to $\pi_{\varphi}(\mathscr{A})^{\prime \prime}$. Then,

$$
\bar{\psi}\left(e^{i t\left(H-H_{\Lambda}-B_{\Lambda}\right)}\right)=\varphi\left(e^{i t\left(H-H_{\Lambda}-B_{\Lambda}\right)}\right) .
$$

Consider the derivative of the above equation and

$$
\varphi(H)=\left(H \Omega_{\varphi}, \Omega_{\varphi}\right)=0, \quad \bar{\psi}(H) \geqq 0 .
$$

As a result,

$$
-\varphi\left(H_{\Lambda}+B_{\Lambda}\right)=\varphi\left(H-H_{\Lambda}-B_{\Lambda}\right)=\bar{\psi}\left(H-H_{\Lambda}-B_{\Lambda}\right) \geqq-\bar{\psi}\left(H_{\Lambda}+B_{\Lambda}\right) .
$$

This gives us (3.8) when $\varphi$ is $\Theta$ invariant.

When the ground state $\varphi$ is not $\Theta$ invariant, consider the $\Theta$ invariant ground state $\varphi \circ p$, where $p$ is the projection to $\mathscr{A}_{+}$defined by

$$
p(Q)=1 / 2\{Q+\Theta(Q)\} .
$$

Take a state $\psi$ satisfying (3.9) for $\varphi$. By the previous step of our proof, we have

$$
\varphi\left(H_{\Lambda}+B_{\Lambda}\right)=\varphi \circ p\left(H_{\Lambda}+B_{\Lambda}\right) \leqq \psi \circ p\left(H_{\Lambda}+B_{\Lambda}\right)=\psi\left(H_{\Lambda}+B_{\Lambda}\right),
$$

which concludes the first part of the proof of Theorem 3.4.

Next we consider the converse direction (the condition 2 to 1 ). So let $\varphi$ be a state satisfying (3.8). Then we first claim that $\varphi \circ p$ is $\alpha_{t}$ invariant. If $Q=Q^{*} \in$ $\mathscr{A}_{\Lambda} \cap \mathscr{A}_{+},(3.7)$ implies

$$
\varphi \circ p\left(e^{i t Q}\left(H_{\Lambda}+B_{\Lambda}\right) e^{-i t Q}\right) \geqq \varphi \circ p\left(H_{\Lambda}+B_{\Lambda}\right)
$$

for any real $t$. Hence by taking the derivative of the above inequality, we get

$$
\varphi \circ p([H, Q])=\varphi \circ p\left(\left[H_{\Lambda}+B_{\Lambda}, Q\right]\right)=0
$$

for $Q$ in $Q=Q^{*} \in \mathscr{A}_{\Lambda} \cap \mathscr{A}_{+}$. By $\Theta$ invariance of $\varphi \circ p$, (3.15) is valid for any $Q \in \mathscr{A}_{\text {loc }}$.

Fix local observables $Q_{+}$in $\mathscr{A}_{+} \cap \mathscr{A}_{\text {loc }}$ and $Q_{-}$in $\mathscr{A}_{-} \cap \mathscr{A}_{\text {loc }}$. Define operators $L_{+}$and $L_{-}$via the equation,

$$
\begin{aligned}
& L_{+}(R)=Q_{+}{ }^{*} R Q_{+}-1 / 2\left\{Q_{+}{ }^{*} Q_{+}, R\right\}, \\
& L_{-}(R)=Q_{-}{ }^{*} \Theta(R) Q_{-}-1 / 2\left\{Q_{-}{ }^{*} Q_{-}, R\right\} .
\end{aligned}
$$


The states $\varphi \circ p \circ e^{t L_{ \pm}}$satisfy the condition (3.9) so

$$
\begin{gathered}
\varphi \circ p\left(e^{t L_{ \pm}}\left(H_{\Lambda}+B_{\Lambda}\right)\right) \geqq \varphi \circ p\left(H_{\Lambda}+B_{\Lambda}\right) \\
\varphi \circ p\left(L_{ \pm}\left(H_{\Lambda}+B_{\Lambda}\right)\right) \geqq 0 .
\end{gathered}
$$

Combined with (3.15), we arrive at

$$
\begin{aligned}
\varphi \circ & p\left(Q_{ \pm}{ }^{*}\left(H_{\Lambda}+B_{\Lambda}\right) Q_{ \pm}-1 / 2\left\{Q_{ \pm}{ }^{*} Q_{ \pm}, H_{\Lambda}+B_{\Lambda}\right\}\right) \\
= & \varphi \circ p\left(Q_{ \pm}^{*}\left(H_{\Lambda}+B_{\Lambda}\right) Q_{ \pm}-1 / 2\left\{Q_{ \pm}{ }^{*} Q_{ \pm}, H_{\Lambda}+B_{\Lambda}\right\}\right) \\
& -1 / 2 \varphi \circ p\left(\left[Q_{ \pm}{ }^{*} Q_{ \pm}, H_{\Lambda}+B_{\Lambda}\right]\right) \\
= & \varphi \circ p\left(Q_{ \pm}^{*}\left[H_{\Lambda}+B_{\Lambda}, Q_{ \pm}\right]\right) \geqq 0 .
\end{aligned}
$$

As the state $\varphi \circ p$ is $\Theta$ invariant the above inequality tells us

$$
\varphi \circ p\left(\left(Q^{*}\left[H_{\Lambda}+B_{\Lambda}, Q\right]\right) \geqq 0\right.
$$

for any $Q=Q_{+}+Q_{-}\left(Q \in \mathscr{A}_{\text {loc }}, Q_{ \pm} \in \mathscr{A}_{\text {loc }} \cap \mathscr{A}_{ \pm}\right)$. So $\varphi \circ p$ is a ground state and so is $\varphi$ due to Proposition 3.2.(ii).

Next we consider translationally invariant states. We show that the translationally invariant ground state minimizes the energy per volume.

Theorem 3.5. We assume the same conditions as those of Theorem 3.4. Futhermore we assume

$$
\lim _{\Lambda \rightarrow \mathbf{Z}^{d}} \frac{\left\|B_{\Lambda}\right\|}{|\Lambda|}=0
$$

If $\varphi$ is a translationally invariant state of $\mathscr{A}$, the following conditions are equivalent:

1. $\varphi$ is a translationally invariant ground state.

2.

$$
\lim _{\Lambda \rightarrow \mathbf{Z}^{d}} \frac{\varphi\left(H_{\Lambda}\right)}{|\Lambda|}=\inf \left\{\lim _{\Lambda \rightarrow \mathbf{Z}^{d}} \frac{\psi\left(H_{\Lambda}\right)}{|\Lambda|}\right\},
$$

where inf is taken for all the translationally invariant states $\psi$.

The statement of Theorem 3.5 is essentially the same as Theorem 1.2. The translationally invariant Hamiltonian of (1.9) and (1.10) is related to $H_{\Lambda}$ as follows. For $h$ satisfying (1.9) and (1.10) we set

$$
\Psi(X)=\sum_{j \in \mathbf{Z}^{d}} \sum_{A=(X, \pm)} h(A) b(A+j) .
$$

It is easy to see that $\lim _{\Lambda \rightarrow \mathbf{Z}^{d}} H_{\Lambda}$ and $H$ of (1.8) gives rise to the same time evolution,

$$
[H, Q]=\lim _{\Lambda \rightarrow \mathbf{Z}^{d}}\left[H_{\Lambda}, Q\right]=\lim _{\Lambda \rightarrow \mathbf{Z}^{d}}\left[H_{\Lambda}+B_{\Lambda}, Q\right]
$$


where $Q$ is in $\mathscr{A}_{\text {loc }}$ and the limit of $\Lambda$ is taken for $d$ dimensional cubes. Due to assumption (1.10), we can also show

$$
\sum_{X: X \ni 0}\|\Psi(X)\| \leqq \sum_{A}|h(A)||A|<\infty .
$$

This leads us to (3.9). Due to the translational invariance, we have also

$$
\lim _{\Lambda \rightarrow \mathbf{Z}^{d}} \frac{\omega\left(H_{\Lambda}\right)}{|\Lambda|}=\omega(h)
$$

for any translationally invariant state $\omega$. Conversely, if a translationally invariant interaction $\Psi=\{\Psi(X)\}$ is given, set

$$
h^{\Psi}=\sum_{X \subset \mathbf{Z}^{d}: X \ni 0} \frac{\Psi(X)}{|X|} .
$$

Then for any translationally invariant state $\omega$, we have $\omega(\Psi(X+j))=\omega(\Psi(X)$ and

$$
\lim _{\Lambda \rightarrow \mathbf{Z}^{d}}\left[H_{\Lambda}, Q\right]=\sum_{j \in \mathbf{Z}^{d}}\left[\tau_{j}\left(h^{\Psi}\right), Q\right]
$$

for any $Q \in \mathscr{A}_{\text {loc }}$. Thus (3.18) can be rewritten as follows:

$$
\varphi\left(h^{\Psi}\right)=\inf \omega\left(h^{\Psi}\right)
$$

where inf is taken for all translationally invariant states $\omega$.

Proof of Theorem 3.5. First we assume that $\varphi$ is a translationally invariant ground state and another translationally invariant state $\psi$ is given. It suffices to show that the $\Theta$ invariant ground state $\varphi \circ p$ satisfies the condition (3.19).

Let $\Lambda$ be a $d$ dimensional cube in $\mathbf{Z}^{d}$. The $C^{*}$-algebra $\mathscr{A}$ is isomorphic to the tensor product of $\mathscr{A}_{\Lambda}$ and its commutant $\mathscr{A}_{\Lambda}^{c}$. Consider the state $\psi_{\Lambda}$ determined by

$$
\psi_{\Lambda}\left(Q_{1} Q_{2}\right)=\varphi \circ p\left(Q_{1}\right) \psi\left(Q_{2}\right)
$$

for $Q_{1} \in \mathscr{A}_{\Lambda}^{c}$ and $Q_{2} \in \mathscr{A}_{\Lambda}$. $\psi_{\Lambda}$ satisfies condition (3.9) for the ground state $\varphi \circ p$ because of Lemma 2.1. We apply Theorem 3.4 now:

$$
\frac{1}{|\Lambda|} \varphi \circ p\left(H_{\Lambda}\right) \leqq \frac{1}{|\Lambda|}\left\{\psi_{\Lambda}\left(H_{\Lambda}+B_{\Lambda}\right)-\varphi \circ p\left(B_{\Lambda}\right)\right\} \leqq \frac{1}{|\Lambda|}\left\{\psi_{\Lambda}\left(H_{\Lambda}\right)+2\left\|B_{\Lambda}\right\|\right\} .
$$

By our assumption (3.18), we obtain (3.19).

Conversely, suppose that $\varphi$ is a state satisfying condition (3.19) of Theorem 3.5. Again we consider the $\Theta$ invariant state $\varphi \circ p$. Thus for $h^{\Psi}$ of (3.24) we have

$$
\varphi \circ p\left(h^{\Psi}\right) \leqq \psi\left(h^{\Psi}\right),
$$

where $\psi$ is an arbitrary translationally invariant state $\psi$. Take a selfadjoint element $Q=Q^{*}$ in $\mathscr{A}_{\text {loc }} \cap \mathscr{A}_{+}$. Set

$$
H^{Q}=\sum_{j} \tau_{j}(Q), \quad \alpha_{t}^{Q}(A)=e^{i t H^{Q}} A e^{-i t H^{Q}} .
$$


As the state $\varphi \circ p f \circ \alpha_{t}^{Q}$ is translationally invariant, $\varphi \circ p\left(\alpha_{t}^{Q}\left(h^{\Psi}\right)\right) \geqq \varphi \circ p\left(h^{\Psi}\right)$. By differentiating this, we have

$$
\begin{aligned}
\sum_{j} \varphi \circ p\left(\left[\tau_{j}(Q), h^{\Psi}\right]\right) & =\sum_{j} \varphi \circ p\left(\tau_{j}\left(\left[Q, \tau_{-j}\left(h^{\Psi}\right)\right]\right)\right) \\
& =\sum_{j} \varphi \circ p\left(\left[Q, \tau_{j}\left(h^{\Psi}\right)\right]\right)=\varphi \circ p([Q, H]) \geqq 0 .
\end{aligned}
$$

If we consider $-Q$ we get $\varphi \circ p([Q, H]) \leqq 0$ and for any $Q$ in $\mathscr{A}_{\text {loc }}$,

$$
\varphi \circ p([Q, H])=0, \quad \varphi \circ p \circ \alpha_{t}=\varphi \circ p
$$

This time invariance is valid for any $Q$ as our state $\varphi \circ p$ is $\Theta$ invariant. Next take another (not neccesarily selfadjoint) element $Q$ in $\mathscr{A}_{\text {loc }} \cap \mathscr{A}_{+}$. Consider the Feller generator $L$ of (2.20) with $E(A)=Q_{+}{ }^{*} A Q_{+}$. Let $S_{t}$ be the Feller semigroup generated by this $L$. Again $\varphi \circ p \circ S_{t}$ is translationally invariant and we have $\varphi \circ p\left(L\left(h^{\Psi}\right)\right) \geqq 0$ as above. By this inequality and the translational invariance of $\varphi \circ p$ we arrive at

$$
\sum_{j} \varphi \circ p\left(Q_{+}{ }^{*} \tau_{j}\left(h^{\Psi}\right) Q_{+}-1 / 2\left\{Q_{+}{ }^{*} Q_{+}, \tau_{j}\left(h^{\Psi}\right)\right\}\right)=\varphi \circ p\left(Q_{+}{ }^{*}\left[H, Q_{+}\right]\right) \geqq 0
$$

where we used the time invariance of $\varphi \circ p$. Take $Q_{-}$from $\mathscr{A}_{\text {loc }} \cap \mathscr{A}_{-}$. Again consider the Markov semigroup $S_{t}$ of (2.20) with $E(A)=Q_{-}{ }^{*} \Theta(A) Q_{-}$. We can proceed as before and obtain (3.27) for $Q_{-}$in place of $Q_{+}$. Thus $\varphi \circ p$ is a ground state. Due to Proposition 3.2 (ii) $\varphi$ is a ground state as well.

\section{Ground States for $\mathscr{A}^{U(1)}$}

In this section, we consider ground states for $\mathscr{A}^{U(1)}$. We present here results corresponding to Theorem 3.4 and 3.5 for $\mathscr{A}^{U(1)}$. In what follows we always assume the conditions of Theorem 3.4 and the gauge invariance of the local Hamiltonian,

$$
\gamma_{\theta}\left(H_{\Lambda}\right)=H_{\Lambda}, \quad \gamma_{\theta}\left(B_{\Lambda}\right)=B_{\Lambda} \quad \text { for any } \theta .
$$

So the time evolution $\alpha_{t}(Q)=\lim _{\Lambda} e^{i t H_{\Lambda}} Q e^{-i t H_{\Lambda}}$ commutes with the gauge transformation $\alpha_{t} \circ \gamma_{\theta}=\gamma_{\theta} \circ \alpha_{t}$. Consider the density operator $n_{A}$ of (1.15). Let $P_{\Lambda}(s)$ be the projection to the eigenvectors of $n_{\Lambda}$ with the eigenvalue $s$. Obviously the possible values of $s$ are $0,1 / \Lambda, \ldots, 1$. By definition of $P_{\Lambda}(s, \delta)$, we have

$$
P_{\Lambda}(s, \delta)=\sum_{s^{\prime}:\left|s^{\prime}-s\right|<\delta} P_{\Lambda}\left(s^{\prime}\right)
$$

Proposition 4.1. Suppose that the translationally invariant interaction $\{\Psi(X)\}$ is gauge invariant, i.e. $\Psi(X) \in \mathscr{A}^{U(1)} \cap \mathscr{A}_{X}$ for any finite subset $X$ of $\mathbf{Z}^{d}$. We assume that the conditions of Theorem 3.4 and Theorem 3.5 are valid. Let $\varphi$ be a state 
of $\mathscr{A}^{U(1)}$. The following conditions are equivalent:

1. $\varphi$ is a ground state of $\mathscr{A}^{U(1)}$.

2. For any $\Lambda$,

$$
\varphi\left(H_{\Lambda}+B_{A}\right)=\inf \left\{\psi\left(H_{A}+B_{A}\right)\right\},
$$

where inf is taken for states $\psi$ of $\mathscr{A}^{U(1)}$ satisfying

$$
\psi\left(Q P_{\Lambda}(s)\right)=\varphi\left(Q P_{\Lambda}(s)\right)
$$

for all $Q \in \mathscr{A}^{U(1)} \cap \mathscr{A}_{\Lambda^{c}}$ and all $s=0,1 / \Lambda, 2 / \Lambda \cdots 1$.

Lemma 4.2. Let $\Lambda$ be a finite subset of $\mathbf{Z}^{d}$. The commutant of $\mathscr{A}^{U(1)} \cap \mathscr{A}_{\Lambda}$ in $\mathscr{A}^{U(1)}$ is generated by $n_{\Lambda}$ of $(1.15)$ and $\mathscr{A}^{U(1)} \cap \mathscr{A}_{\Lambda^{c}}$.

Proof of Lemma 4.2. Suppose that $Q$ in $\mathscr{A}^{U(1)}$ commutes with $\mathscr{A}^{U(1)} \cap \mathscr{A}_{\Lambda}$,

$$
[Q, D]=0 \quad D \text { in } \mathscr{A}^{U(1)} \cap \mathscr{A}_{\Lambda} .
$$

Then $Q$ can be written in the following form:

$$
Q=\sum_{F, G \subset A} Q_{F G} \prod_{F \ni l} a_{l}^{*} \prod_{G \ni k} a_{k},
$$

where $Q_{F G}$ is in $\mathscr{A}_{\Lambda^{c}}$. For any site $j$ in $\Lambda$ we have

$$
Q=\int_{0}^{2 \pi} e^{i \theta n_{j}} Q e^{-i \theta n_{j}} d \theta .
$$

On the other hand,

$$
\int_{0}^{2 \pi} e^{i \theta n_{\jmath}} a_{k} e^{-i \theta n_{\jmath}} d \theta=\left\{\begin{array}{ll}
0 & \text { if } k=j \\
a_{k} & \text { if } k \neq j
\end{array} .\right.
$$

Thus the summand of (4.3) does vanish when $j \in F \cap G^{c}$ and when $j \in F^{c} \cap G$. As $j$ is an arbitrary site in $\Lambda$, we can conclude that $Q_{F G}=0$ unless $F=G$. So

$$
Q=\sum_{A \subset \Lambda} Q_{F F} \prod_{F \ni l} a_{l}^{*} \prod_{F \ni k} a_{k}=\sum_{F \subset \Lambda} Q_{F}^{\prime} \prod_{F \ni l} n_{l},
$$

where $Q_{F}^{\prime}$ is in $\mathscr{A} \cap \mathscr{A}_{\Lambda^{c}}$. Due to the gauge invariance of $Q, Q_{F}^{\prime}$ belongs to $\mathscr{A}^{U(1)} \cap \mathscr{A}_{\Lambda^{c}}$.

On the other hand, the permutation of lattice sites $i$ and $j$ in $\Lambda$ gives rise to the inner automorphism $\Xi_{i j}$. It is possible to show that $\Xi_{i j}$ is implemented by a selfadjoint unitary of $\mathscr{A}^{U(1)} \cap \mathscr{A}_{\Lambda}$. As a result, $Q$ is invariant under $\Xi_{i j}$. By averaging via the Haar measure of the permutation group of lattice sites in $\Lambda, Q$ can be expressed in the following way:

$$
Q=\sum_{\alpha=1,2,, m} Q_{\alpha} B_{\alpha},
$$

where $Q_{\alpha}$ belongs to $\mathscr{A}^{U(1)} \cap \mathscr{A}_{\Lambda^{c}}, B_{\alpha}$ belongs to $\mathscr{B} \cap \mathscr{A}_{\Lambda}$ and $\Xi_{i j}\left(B_{\alpha}\right)=B_{\alpha}$ for any $i, j$ in $\Lambda$. Recall that we defined $\mathscr{B}$ as the abelian algebra generated by number operators in Sect. 1 so $\mathscr{B} \cap \mathscr{A}_{\Lambda}$ is the abelian algebra generated by $n_{j}(j \in \Lambda)$. 
To complete our proof, we have only to show that any permutation invariant element of $\mathscr{B} \cap \mathscr{A}_{\Lambda}$ is a polynomial of $n_{\Lambda}$. As $n_{j}{ }^{2}=n_{j}$, any elementary symmetric polynomial of $n_{J}(j \in \Lambda)$ is a polynomial of $n_{\Lambda}$.

Lemma 4.2 tells us that the commutant of $\mathscr{A}^{U(1)} \cap \mathscr{A}_{1}$ in $\mathscr{A}^{U(1)}$ is the direct sum of $|\Lambda|+1$ copies of $\mathscr{A}^{U(1)} \cap \mathscr{A}_{\Lambda^{c}}$,

$$
\left(\mathscr{A}^{U(1)} \cap \mathscr{A}_{\Lambda}\right)^{c} \cap \mathscr{A}^{U(1)}=\sum \mathscr{A}^{U(1)} \cap \mathscr{A}_{1}^{c} \otimes \mathbf{C},
$$

where each summand corresponds to the center $P_{\Lambda}(s)\left(\mathscr{A}^{U(1)} \cap \mathscr{A}_{\Lambda}\right) P_{\Lambda}(s)$ of $\mathscr{A}^{U(1)}$ $\cap \mathscr{A}_{\Lambda}$.

It is possible to show that the state $\psi$ satisfying (4.1) is quasi-equivalent to $\varphi$ and the proof of Proposition 4.1 is essentially same as that of Theorem 6 of [4]. We do not repeat it here.

Next, we consider translationally invariant ground states for $\mathscr{A}^{U(1)}$. Due to Proposition 4.3 below, it suffices to consider states in the fixed density $s$ of particles in the sense of (1.19). Now we fix the particle density $s$ with $0<s<1$. The states $\varphi$ with no particle $s=0$ or no antiparticle $s=1$ are the Fock states characterized by the equations, $\varphi\left(n_{j}\right)=0$ (or $\varphi\left(n_{j}\right)=1$ ) for any $j$ in $\mathbf{Z}^{d}$. These are not interesting in our context and we will not mention these cases.

Proposition 4.3. Let $\psi$ be a translationally invariant state of $\mathscr{A}^{U(1)}$ with $\psi\left(n_{j}\right)=s$. Assume that the restriction of $\psi$ to $\mathscr{B}$ is an ergodic measure with respect to the translation (the shift on $\mathbf{Z}^{d}$ ). Then it is a state with the density $s$ in the sense of Defintion 1.3. In particular, any ergodic state of $\mathscr{A}^{U(1)}$ is a state with the density s.

Note that the restriction of an ergodic state of $\mathscr{A}^{U(1)}$ or $\mathscr{A}$ to $\mathscr{B}$ is an ergodic measure.

Proof of Proposition 4.3. Recall that $\mathscr{B}$ is an abelian $C^{*}$-algebra generated by number operators $n_{J}$. Consider the measure on $\{1,0\}^{Z^{d}}$ induced by the restriction of $\psi$ to $\mathscr{B}$. Due to our assumption of ergodicity, the function $n_{A}=n_{A}(x)$ converges to the constant $s$ almost everywhere,

$$
\lim _{\Lambda \rightarrow \mathbf{Z}^{d}} n_{\Lambda}(x)=\psi\left(n_{j}\right)=s \quad \text { (a.e.). }
$$

Let us prove (1.19). Take $\delta_{1}$ and $\delta_{2}$ satisfying $0<\delta_{1}<\delta_{2}<\delta$ and a continuous function $\chi(t)$ on $R$ such that

$$
0 \leqq \chi \leqq 1, \quad \chi(t)= \begin{cases}1, & \text { if }|t-s|<\delta_{1} \\ 0, & \text { if }|t-s|>\delta_{2}\end{cases}
$$

Then

$$
0 \leqq \chi\left(n_{\Lambda}(x)\right) \leqq P_{\Lambda}(s, \delta) \leqq 1
$$

Due to (4.1) we have

$$
\lim _{\Lambda \rightarrow \mathbf{Z}^{d}} \chi\left(n_{\Lambda}(x)\right)=\chi(s)=1 \quad \text { (a.e.) }
$$

and

This implies (1.19).

$$
\lim _{\Lambda \rightarrow \mathbf{Z}^{d}} P_{\Lambda}(s, \delta)=1 \quad \text { (a.e.). }
$$


Remark 4.4. (i) Even though the state $\varphi$ is not ergodic, we have the following convergence in the strong operator topology in the GNS representation space:

$$
\lim _{\Lambda \rightarrow \mathbf{Z}^{d}} \pi_{\varphi}\left(n_{\Lambda}\right)=n_{\infty}
$$

In fact, the above limit exists almost everywhere due to the Ergodic theorem. As $\left\|n_{\Lambda}\right\|=1$, the convergence is in $L^{2}\left(\{0,1\}^{\mathbf{Z}^{d}}\right)$. So

$$
\lim _{\Lambda \rightarrow \mathbf{Z}^{d}}\left\|\left(\pi_{\varphi}\left(n_{\Lambda}\right)-n_{\infty}\right) \Omega_{\varphi}\right\|=0 .
$$

For the vector $\pi_{\varphi}(Q) \Omega_{\varphi}$ with $Q \in \mathscr{A}_{\text {loc }}$ we have

$$
\lim _{\Lambda \rightarrow \mathbf{Z}^{d}}\left\|\left(\pi_{\varphi}\left(n_{\Lambda}\right)-n_{\infty}\right) \pi_{\varphi}(Q) \Omega_{\varphi}\right\|=0,
$$

because

$$
\begin{gathered}
\lim _{\Lambda \rightarrow \mathbf{Z}^{d}}\left(\left(\pi_{\varphi}\left(n_{\Lambda}\right)-n_{\infty}\right) \pi_{\varphi}(Q) \Omega_{\varphi},\left(\pi_{\varphi}\left(n_{\Lambda}\right)-n_{\infty}\right) \pi_{\varphi}(Q) \Omega_{\varphi}\right) \\
\lim _{\Lambda \rightarrow \mathbf{Z}^{d}}\left(\pi_{\varphi}\left(Q^{*} Q\right) \Omega_{\varphi},\left(\pi_{\varphi}\left(n_{\Lambda}\right)-n_{\infty}\right)^{2} \Omega_{\varphi}\right)=0 .
\end{gathered}
$$

(ii) Proposition 4.3 tells us how a translationally invariant state $\varphi$ of $\mathscr{A}^{U(1)}$ is decomposed to the states $\varphi_{s}$ with density $s$. For non ergodic $\varphi$ consider the spectral decomposition of $n_{\infty}$ and the spectral measure $\mu$ of the state $\varphi$,

$$
\int_{0}^{1} s d E(s)=n_{\infty}, \quad \mu([0, s])=\bar{\varphi}(E([0, s]),
$$

where $\bar{\varphi}()$ is the normal extension to $\pi\left(\mathscr{A}^{U(1)}\right)^{\prime \prime}$. Note that $n_{\infty}$ is in the centre of the von Neumann Algebra $\pi\left(\mathscr{A}^{U(1)}\right)^{\prime \prime}$. Then the decomposition of $\varphi$ is

$$
\varphi=\int^{\oplus} \varphi_{s} d \mu(s)
$$

Lemma 4.5. A translationally invariant state $\psi$ has the density $s$ if and only if for any positive integer $k$,

$$
\lim _{\Lambda \rightarrow \mathbf{Z}^{d}} \psi\left(n_{\Lambda}{ }^{k}\right)=s^{k} .
$$

The proof follows from the Stone-Weierstrauss Theorem for approximation of continuous functions by polynomials.

Now we restate Theorem 1.4 in a slightly more general situation.

Proposition 4.6. Suppose that the translationally invariant interaction $\{\Psi(X)\}$ is gauge invariant, i.e. $\Psi(X) \in \mathscr{A}^{U(1)} \cap \mathscr{A}_{X}$ for any finite subset $X$ of $\mathbf{Z}^{d}$. We assume the conditions of Theorem 3.4 and

$$
\lim _{\Lambda \rightarrow \mathbf{Z}^{d} X \cap \Lambda \neq \emptyset, X \cap \Lambda^{c} \neq \emptyset} \frac{\|\Psi(X)\|}{|\Lambda|}=0 .
$$

Let $\varphi$ be a translationally invariant state of $\mathscr{A}^{U(1)}$ with the density s. Then the following conditions are equivalent:

1. $\varphi$ is a translationally invariant ground state of $\mathscr{A}^{U(1)}$. 
2.

$$
\lim _{\Lambda \rightarrow \mathbf{Z}^{d}} \frac{\varphi\left(H_{\Lambda}\right)}{|\Lambda|}=\inf \left\{\lim _{\Lambda \rightarrow \mathbf{Z}^{d}} \frac{\psi\left(H_{\Lambda}\right)}{|\Lambda|}\right\},
$$

where inf is taken among all translationally invariant states $\psi$ of $\mathscr{A}^{U(1)}$ with density $s$

We present our proof of Proposition 4.6 after Lemma 4.10.

Remark 4.7. Suppose the assumptions of Theorem 4.3 are valid. For a real number $\mu$ set

$$
H_{\Lambda}(\mu)=H_{\Lambda}+\mu \sum_{k \in \Lambda} n_{j}
$$

Let $\alpha_{t}(\mu)$ be the one-parameter group of automorphisms of $\mathscr{A}$ with the Hamiltonian $H(\mu)=\lim _{\Lambda \rightarrow \mathbf{Z}^{d}} H_{\Lambda}(\mu)$,

$$
\alpha_{t}(\mu)(Q)=e^{t \delta(\mu)}(Q), \quad \delta(\mu)(Q)=i \lim _{\Lambda \rightarrow \mathbf{Z}^{d}}\left[H_{\Lambda}(\mu), Q\right] .
$$

It is easy to see

$$
\alpha_{t}(\mu)(Q)=\alpha_{t} \circ \gamma_{\mu t}(Q) .
$$

Thus any ground state of $\left(\mathscr{A}, \alpha_{t}(\mu)\right)$ yields a ground state for $\left(\mathscr{A}^{U(1)}, \alpha_{t}\right)$ via restriction. We may ask whether any ground state of $\left(\mathscr{A}^{U(1)}, \alpha_{t}\right)$ is obtained in this way. The same question can be posed for KMS states (=Gibbs states) and was solved by Araki and Kishimoto ([1]) for abelian gauge groups and by Araki, Kastler, Haag, Takesaki for general compact gauge groups in [2].

The following Proposition 4.8 is a partial answer to the question in the case of the ground state.

Proposition 4.8. Suppose that the assumptions of Proposition 4.6 are valid. Let $\varphi$ be a translationally invariant ground state of $\left(\mathscr{A}^{U(1)}, \alpha_{t}\right)$ with density $s$ satisfying

$$
0<s=\varphi\left(n_{0}\right)<1 .
$$

Consider the gauge invariant extension $\tilde{\varphi}$ of $\varphi$ to $\mathscr{A}$. Then there exists $\mu$ such that $\tilde{\varphi}$ is a ground state for $\left(\mathscr{A}, \alpha_{t}(\mu)\right)$.

We now begin our proof of Propositions 4.6 and 4.8. We consider the mean ground state energy.

Let $e_{\Lambda}(s)$ be the smallest eigenvalue of $\frac{1}{|\Lambda|} P_{\Lambda}(s) H_{\Lambda}$. Obviously,

$$
|\Lambda| e_{\Lambda}(s)=\inf _{\psi} \frac{\psi\left(P_{\Lambda}(s) H_{\Lambda}\right)}{\psi\left(P_{\Lambda}(s)\right)} .
$$

Proposition 4.9. Suppose the interaction is translationally invariant. Assume also that the conditions of Proposition 4.6 are valid. The following limit exists:

$$
\lim _{\Lambda \rightarrow \mathbf{Z}^{d}} e_{\Lambda}(s)=e(s)
$$

This convergence is uniform for $s$ in any closed interval $[a, b] \subset(0,1)$. e(s) is a convex function of $s$ and for any sequence $s_{n}$ with $\lim s_{n}=s$ and any sequence of cubes $\Lambda_{n}$ with $\lim \Lambda_{n}=\mathbf{Z}^{d}$, we have

$$
\lim _{n \rightarrow \infty} e_{\Lambda_{n}}\left(s_{n}\right)=e(s) .
$$


Sketch of proof of Proposition 4.9. This is somewhat standard in mathematical statistical physics. (See Sect. 3 of [8].) We show the uniform convergence of (4.12) which will be important for the next proposition.

For $\Lambda=\Lambda_{1} \cup \Lambda_{2}, \Lambda_{1} \cap \Lambda_{2}=\emptyset$,

$$
H_{\Lambda}=H_{\Lambda_{1}}+H_{\Lambda_{2}}+\sum_{X \cap \Lambda_{1} \neq \emptyset, X \cap \Lambda_{2} \neq \emptyset} \Psi(X) .
$$

So we have

$$
H_{\Lambda} \leqq H_{\Lambda_{1}}+H_{\Lambda_{2}}+\left(|B|_{\Lambda_{1}}+|B|_{\Lambda_{2}}\right) 1
$$

where we set

$$
|B|_{\Lambda}=\sum_{X \cap A \neq \emptyset, X \cap A^{c} \neq \emptyset}\|\Psi(X)\|
$$

By (4.8), $\lim _{\Lambda} \frac{|B|_{\Lambda}}{|\Lambda|}=0$. By the isomorphism (2.1) of CAR and the tensor algebra of matrices,

$$
H_{\Lambda_{1}}+H_{\Lambda_{2}}=H_{\Lambda_{1}} \otimes 1+1 \otimes H_{\Lambda_{2}},
$$

we can find unit eigenvectors $\psi_{1}$ and $\psi_{2}$ satisfying

$$
H_{\Lambda_{j}} \psi_{j}=\left|\Lambda_{j}\right| e_{\Lambda_{j}}\left(s_{j}\right) \psi_{j}, \quad n_{\Lambda_{j}} \psi_{j}=s_{j} \psi_{j} \quad(j=1,2) .
$$

Consider $\psi=\psi_{1} \otimes \psi_{2}$. When $|\Lambda| s=\left|\Lambda_{1}\right| s_{1}+\left|\Lambda_{2}\right| s_{2}$ we have

$$
\begin{gathered}
\left(H_{\Lambda_{1}}+H_{\Lambda_{2}}\right) \psi=\left(\left|\Lambda_{1}\right| e_{\Lambda_{1}}(s)+\left|\Lambda_{2}\right| e_{\Lambda_{2}}(s)\right) \psi, \\
n_{\Lambda} \psi=s \psi .
\end{gathered}
$$

As a result,

$$
|\Lambda| e_{\Lambda}(s) \leqq\left|\Lambda_{1}\right| e_{\Lambda_{1}}\left(s_{1}\right)+\left|\Lambda_{2}\right| e_{\Lambda_{2}}\left(s_{2}\right)+\left(|B|_{\Lambda_{1}}+|B|_{\Lambda_{2}}\right) .
$$

By the same reason, when $\Lambda^{\prime}$ is the disjoint union of $k$ translates of $\Lambda$ and the remainder $\Lambda^{\prime \prime}$ we have

$$
\left|\Lambda^{\prime}\right| e_{\Lambda^{\prime}}(s) \leqq k|\Lambda| e_{\Lambda}(s)+\left|\Lambda^{\prime \prime}\right| e_{\Lambda^{\prime \prime}}(s)+\left(k\left(|B|_{\Lambda}+|B|_{\Lambda^{\prime \prime}}\right)\right) .
$$

By our assumption (3.3) we have a positive constant $M$ such that

$$
e_{\Lambda}(s) \leqq M
$$

independent of $s$ and $\Lambda$. Due to (3.18), we can find a cube $\Lambda_{0}$ such that the following is valid when $\Lambda \supset \Lambda_{0}$ :

$$
\frac{|B|_{\Lambda}}{|\Lambda|} \leqq \varepsilon
$$

Next we show the convergence of (4.12) for $\Lambda(k)=\left[(-2)^{k}, 2^{k}\right]^{d}$. Then the remainder term of $\Lambda^{\prime \prime}$ is absent in (4.15). So when $\Lambda_{0} \subset \Lambda\left(k_{0}\right)$ and $l>k>k_{0}$,

$$
e_{\Lambda(l)}(s) \leqq e_{\Lambda(k)}(s)+\varepsilon .
$$

Set

$$
\bar{e}_{k}(s)=\sup _{j: j \geqq k} e_{\Lambda(j)}(s), \quad \bar{e}(s)=\lim _{k \rightarrow \infty} \bar{e}_{k}(s) .
$$


Equation (4.17) tells us

$$
\bar{e}_{k}(s) \leqq e_{\Lambda(k)}(s)+\varepsilon \leqq \bar{e}_{k}(s)+\varepsilon,
$$

and for $k>k_{1}=k_{1}(s)$ we have

$$
\left|e_{\Lambda(k)}(s)-\bar{e}(s)\right|<2 \varepsilon,
$$

which implies the convergence,

$$
\lim _{k} e_{\Lambda(k)}(s)=\bar{e}(s) .
$$

The convergence (4.12) can be shown by estimation of $\Lambda^{\prime \prime}$ in (4.15) and the convexity follows from the approximate convexity of $e_{\Lambda}(s)$ in (4.13),

$$
e_{\Lambda}(s) \leqq \lambda e_{\Lambda_{1}}\left(s_{1}\right)+(1-\lambda) e_{\Lambda_{2}}\left(s_{2}\right)+\frac{\varepsilon}{|\Lambda|}
$$

with $\lambda=\frac{\left|\Lambda_{1}\right|}{|\Lambda|}, 1-\lambda=\frac{\left|\Lambda_{2}\right|}{|\Lambda|}$.

The approximate convexity and the uniform boundedness (4.16) also imply equicontinuity of $e_{\Lambda}(s)$. As $e(s)$ is convex and bounded, it is continuous. Thus by equicontinuity of $e_{\Lambda}(s)$ and continuity of $e(s)$, uniform convergence of $\lim e_{\Lambda}(s)=$ $e(s)$ is implied by the $3 \varepsilon$ argument.

Lemma 4.10. We assume the conditions of Proposition 4.6. Let $\varphi$ be a translationally invariant ground state of $\mathscr{A}^{U(1)}$ with density $s$. Then,

$$
\lim _{\Lambda \rightarrow \mathbf{Z}^{d}} \frac{\varphi\left(H_{\Lambda}\right)}{|\Lambda|}=e(s) .
$$

For any translationally invariant state $\psi$ with density $s$, we have

$$
e(s) \leqq \lim _{\Lambda \rightarrow \mathbf{Z}^{d}} \frac{\psi\left(H_{\Lambda}\right)}{|\Lambda|} \text {. }
$$

Proof of Lemma 4.10 We use Proposition 4.1. Let $\varphi$ be a ground state of $\mathscr{A}^{U(1)}$ with density $s$. Then due to Proposition 4.1 , if $\Lambda$ is sufficiently large,

$$
\frac{1}{|\Lambda|} \varphi\left(H_{\Lambda}+B_{\Lambda}\right) \leqq \sum_{s^{\prime}} \varphi\left(P_{\Lambda}\left(s^{\prime}\right)\right) e_{\Lambda}\left(s^{\prime}\right)+\varepsilon .
$$

By (1.19),

$$
\begin{aligned}
\varphi\left(P_{\Lambda}(s, \delta)\right)= & \sum_{\left|s^{\prime}-s\right|<\delta} \varphi\left(P_{\Lambda}\left(s^{\prime}\right)\right)>1-\varepsilon, \\
& \sum_{\left|s^{\prime}-s\right| \geqq \delta} \varphi\left(P_{\Lambda}\left(s^{\prime}\right)\right) \leqq \varepsilon .
\end{aligned}
$$


Thus

$$
\begin{aligned}
\lim _{\Lambda \rightarrow \mathbf{Z}^{d}} \frac{1}{|\Lambda|} \varphi\left(H_{\Lambda}\right) & =\lim _{\Lambda \rightarrow \mathbf{Z}^{d}} \frac{1}{|\Lambda|} \varphi\left(H_{\Lambda}+B_{\Lambda}\right) \\
& \leqq \lim _{\Lambda \rightarrow \mathbf{Z}^{d}} \sum_{\left|s^{\prime}-s\right|<\delta} \varphi\left(P_{\Lambda}\left(s^{\prime}\right)\right) e_{\Lambda}\left(s^{\prime}\right)+2 \varepsilon \\
& \leqq \lim _{\Lambda \rightarrow \mathbf{Z}^{d}} \sup _{\left|s^{\prime}-s\right|<\delta}\left(e_{\Lambda}\left(s^{\prime}\right)\right)+2 \varepsilon \\
& \leqq \sup _{\left|s^{\prime}-s\right|<\delta}\left(e\left(s^{\prime}\right)\right)+2 \varepsilon .
\end{aligned}
$$

This implies (4.18).

Let $\psi$ be a state of $\mathscr{A}^{U(1)}$ with density $s$. Then

$$
\begin{aligned}
\frac{1}{|\Lambda|} \psi\left(H_{\Lambda}\right) & \geqq \sum_{s^{\prime}} \psi\left(P_{\Lambda}\left(s^{\prime}\right)\right) e_{\Lambda}\left(s^{\prime}\right) \\
& \geqq \sum_{\left|s^{\prime}-s\right|<\delta} \psi\left(P_{\Lambda}\left(s^{\prime}\right)\right) e_{\Lambda}\left(s^{\prime}\right)-\varepsilon \\
& \geqq(1-\varepsilon) \inf _{\left|s^{\prime}-s\right|<\delta}\left(e_{\Lambda}\left(s^{\prime}\right)\right)-\varepsilon .
\end{aligned}
$$

This implies (4.19).

Proof of Proposition 4.6. Due to Lemma 4.10, it suffices to show $2 \Rightarrow 1$. So we assume that $\varphi$ satisfies condition 2. We again use the Markov semigroup technique of our proof of Theorem 3.5. Let $Q=Q^{*}$ be an element of $\mathscr{A}^{U(1)} \cap \mathscr{A}_{\text {loc }}$ and consider $\alpha_{t}^{Q}$ of (3.26). If we show the state $\varphi \circ \alpha_{t}^{Q}$ has the density $s$, condition 2 of Proposition 4.6 implies that $\varphi$ is invariant under the time evolution $\alpha_{t}$. We now show that $\varphi \circ \alpha_{t}^{Q}$ has the density $s$. Due to Lemma 4.5, we have only to show

$$
\lim _{\Lambda \rightarrow \mathbf{Z}^{d}} \varphi \circ \alpha_{t}^{Q}\left(n_{\Lambda}^{k}\right)=s^{k}
$$

In fact,

$$
\varphi \circ \alpha_{t}^{Q}\left(n_{\Lambda}{ }^{k}\right)=\varphi\left(n_{\Lambda}{ }^{k}\right)+\int_{0}^{t} \varphi \circ \alpha_{u}^{Q}\left(\left[H_{Q}, n_{\Lambda}{ }^{k}\right]\right) d u
$$

However,

$$
\left[H_{Q}, n_{\Lambda}{ }^{k}\right]=\sum_{j}\left[\tau_{j}(Q), n_{\Lambda}{ }^{k}\right]
$$

As $\tau_{j}(Q)$ is gauge invariant $\left[\tau_{j}(Q), n_{\Lambda}{ }^{k}\right]=0$ unless $j$ is near the boundary of $\Lambda$. So we have a constant $C$ such that

$$
\left|\left[H_{Q}, n_{\Lambda}{ }^{k}\right]\right| \leqq C k \frac{|\partial \Lambda|}{|\Lambda|}|\operatorname{support}(Q)|\|Q\|
$$

In the thermodynamic limit,

$$
\lim _{\Lambda \rightarrow \mathbf{Z}^{d}} \varphi \circ \alpha_{t}^{Q}\left(n_{\Lambda}^{k}\right)=\lim _{\Lambda \rightarrow \mathbf{Z}^{d}} \varphi\left(n_{\Lambda}{ }^{k}\right)=s^{k} .
$$


Next we show that $\varphi$ is a ground state. Take $Q \in \mathscr{A}^{U(1)} \cap \mathscr{A}_{\text {loc }}$ and consider the Markov semigroup $S_{t}$ generated by $L$ of (2.20) with $E(A)=Q^{*} A Q$. Then as is the case of $\varphi \circ \alpha_{t}^{Q}$, the state $\varphi \circ S_{t}$ has the density $s$ and

$$
\varphi \circ S_{t}\left(h^{\Psi}\right) \geqq \varphi\left(h^{\psi}\right), \quad \varphi\left(L\left(h^{\Psi}\right)=\varphi\left(Q^{*}[H, Q]\right) \geqq 0,\right.
$$

which suggests that $\varphi$ is a ground state.

For our proof of Proposition 4.8 we need some more preparation. For a translationally invariant state $\varphi$ of $\mathscr{A}$ or $\mathscr{A}^{U(1)}$, we set

$$
s(\varphi)=\varphi\left(n_{0}\right) \quad\left(=\varphi\left(n_{j}\right) \text { for any } j\right) .
$$

We also define $E(\mu)$ as the mean ground state energy (3.25) of $H(\mu)$, so for a translationally invariant ground state $\varphi_{\mu}$ of $\left(\mathscr{A}, \alpha_{t}(\mu)\right)$,

$$
E(\mu)=\varphi_{\mu}\left(h^{\Psi}\right)+\mu s\left(\varphi_{\mu}\right)
$$

Lemma 4.11. Let $\varphi_{\mu}$ be a translationally invariant ground state of $\left(\mathscr{A}, \alpha_{t}(\mu)\right)$. Then,

$$
e\left(s\left(\varphi_{\mu}\right)\right) \leqq E(\mu)-\mu s\left(\varphi_{\mu}\right) .
$$

Proof of Lemma 4.11. Consider the restriction of $\varphi_{\mu}$ to $\mathscr{A}^{U(1)}$ which is a ground state for $\left(\mathscr{A}^{U(1)}, \alpha_{t}\right)$. The decomposition of $\varphi_{\mu}$ to the states $\varphi(s)$ with density $s$ gives rise to that of ground states for $\left(\mathscr{A}^{U(1)}, \alpha_{t}\right)$ (cf. Remark 4.4 and the proof of Proposition 4.3)

$$
\varphi_{\mu}=\int \varphi(s) d v(s)
$$

Thus by (4.18) and the convexity of $e(s)$ we have

$$
\begin{aligned}
e\left(s\left(\varphi_{\mu}\right)\right. & =e\left(\int s d v(s)\right) \leqq \int e(s) d v(s) \\
& =\int \varphi(s)\left(h^{\Psi}\right) d v(s)=\varphi_{\mu}\left(h^{\Psi}\right) \\
& =E(\mu)-\mu s\left(\varphi_{\mu}\right) . \quad \square
\end{aligned}
$$

Lemma 4.12. Let $\varphi$ be an ergodic ground state for $\left(\mathscr{A}^{U(1)}, \alpha_{t}\right)$. Suppose there exists a translationally invariant ground state $\varphi_{\mu}$ for $\left(\mathscr{A}, \alpha_{t}(\mu)\right)$ with the property, $s\left(\varphi_{\mu}\right)=s(\varphi)$. Then the gauge invariant extension $\tilde{\varphi}$ of $\varphi$ to $\mathscr{A}$ is a ground state for $\left(\mathscr{A}, \alpha_{t}(\mu)\right)$.

Proof of Lemma 4.12. Set $s_{0}=s\left(\varphi_{\mu}\right)=s(\varphi)=\varphi\left(n_{0}\right)$. Due to Lemma 4.11, and Theorem 3.5,

$$
\varphi_{\mu}\left(h^{\Psi}\right)=E(\mu)-\mu s_{0} \geqq e\left(s_{0}\right),
$$

so by Proposition 4.6 we have

$$
\tilde{\varphi}\left(h^{\Psi}+\mu n_{0}\right)=\varphi\left(h^{\Psi}\right)+\mu s_{0}=e\left(s_{0}\right)+\mu s_{0} \leqq E(\mu) .
$$


By Theorem 3.5, $\tilde{\varphi}\left(h^{\Psi}+\mu n_{0}\right)$ can not exceed $E(\mu)$, so

$$
\tilde{\varphi}\left(h^{\Psi}+\mu n_{0}\right)=E(\mu),
$$

which tells us that $\tilde{\varphi}$ is a ground state for $\alpha_{t}(\mu)$.

Proof of Proposition 4.8. Due to Lemma 4.12, it suffices to show that for any $s(0<s<1)$ there exists at least one translationally invariant ground state $\psi$ of $\left(\mathscr{A}, \alpha_{t}(\mu)\right)$ with $s=s(\psi)$ for some $\mu$. For any $\mu$ we have at least one translationally invariant ground state, say, $\varphi_{\mu}$ for $\left(\mathscr{A}, \alpha_{t}(\mu)\right)$.

Consider $s\left(\varphi_{\mu}\right)$. We claim that it is non-increasing as a function of $\mu$. To see this recall Theorem 3.5 and Definition (4.25) of $E(\mu)$. Then

$$
\begin{aligned}
E(\mu) & =\varphi_{\mu}\left(h^{\Psi}+\mu n_{0}\right) \leqq \varphi_{\mu^{\prime}}\left(h^{\Psi}+\mu n_{0}\right) \\
& =\varphi_{\mu^{\prime}}\left(h^{\Psi}+\mu^{\prime} n_{0}\right)+\left(\mu-\mu^{\prime}\right) \varphi_{\mu^{\prime}}\left(n_{0}\right)=E\left(\mu^{\prime}\right)+\left(\mu-\mu^{\prime}\right) s\left(\varphi_{\mu^{\prime}}\right),
\end{aligned}
$$

and by interchanging $\mu$ and $\mu^{\prime}$ we have

$$
E\left(\mu^{\prime}\right) \leqq E(\mu)+\left(\mu^{\prime}-\mu\right) s\left(\varphi_{\mu}\right) .
$$

Adding these inequalities (4.30) and (4.31) we obtain

$$
0 \leqq\left(\mu^{\prime}-\mu\right)\left(s\left(\varphi_{\mu}\right)-s\left(\varphi_{\mu^{\prime}}\right)\right)
$$

So we have proved that $s\left(\varphi_{\mu}\right)$ is non-increasing.

The set of discontinuity for $s\left(\varphi_{\mu}\right)$ is at most a countable set. Suppose at $\mu=\mu_{0}$, $s\left(\varphi_{\mu}\right)$ is discontinuous. Set

$$
\begin{aligned}
\lim _{\mu \uparrow \mu_{0}} s\left(\varphi_{\mu}\right)=s_{-}, & & \lim _{\mu \downarrow \mu_{0}} s\left(\varphi_{\mu}\right)=s_{+}, \\
\lim _{\mu \uparrow \mu_{0}} \varphi_{\mu}=\varphi_{-}, & & \lim _{\mu \downarrow \mu_{0}} \varphi_{\mu}=\varphi_{+} .
\end{aligned}
$$

The convex combination $\varphi=t \varphi_{-}+(1-t) \varphi_{+}$is a ground state for $\left(\mathscr{A}, \alpha_{t}\left(\mu_{0}\right)\right)$ and

$$
s(\varphi)=t s\left(\varphi_{-}\right)+(1-t) s\left(\varphi_{+}\right) .
$$

So for any $s$ with $s_{-} \leqq s \leqq s_{+}$, there exists a translationally invariant ground state for $\left(\mathscr{A}, \alpha_{t}(\mu)\right)$.

To complete the proof, we have to show

$$
\lim _{\mu \rightarrow \infty} s\left(\varphi_{\mu}\right)=0, \quad \lim _{\mu \rightarrow-\infty} s\left(\varphi_{\mu}\right)=1 .
$$

Consider the mean ground state energy $E(\mu)$ and the energy expectation of the Fock state $\varphi_{F}\left(\varphi_{F}\left(n_{J}\right)=0\right.$ for all $\left.j\right)$. Then by Theorem 3.5,

$$
\varphi_{\mu}\left(h^{\Psi}\right)+\mu s\left(\varphi_{\mu}\right) \leqq \varphi_{F}\left(h^{\Psi}\right) .
$$

Thus

$$
0 \leqq \mu s\left(\varphi_{\mu}\right) \leqq 2\left\|h^{\Psi}\right\|,
$$

and we obtain the first identity of (4.35). If we take the anti-Fock state $\varphi_{A F}$ $\left(\varphi_{A F}\left(n_{j}\right)=1\right.$ for all $\left.j\right)$ we obtain the second identity of (4.35). 


\section{References}

1 Araki, H, Kishimoto, A: Symmetry and equilibrium states Commun Math Phys 53, 211-232 (1977)

2 Araki, H, Haag, R, Kastler, D, Takesaki, M : Extension of KMS States and Chemical Potential Commun Math. Phys 53, 97-134 (1977)

3 Bratteli, O, Jørgensen, P (eds ): Positive semigroups of operators, and applications Acta Appl. Math 2, Nos 3/4 (1984)

4 Bratteli, O, Kishimoto, A, Robinson, D : Ground states of quantum spin systems Commun Math Phys 64, 41-48 (1978)

5 Bratteli, O, Robinson, D : Operator algebras and quantum statistical mechanics I, II BerlinHeidelberg-New york: Springer, 1979

6 Davies, E B : Quantum Theory of Open Systems London-New York: Academic Press, 1976

7 Davies, E B, Lindsay, M: Superderivations and Symmetric Markov Semigroups Commun Math Phys 157, 359-370 (1993)

8 Georgii, H : Canonical Gibbs Measures Springer Lect Note in Math 760, Berlin-HeidelbergNew York: Springer

9 Georgii, H: Gibbs Measures and Phase Transitions Amsterdam: Walter de Gruyter, 1988

10 Gottstein, C T, Werner, R: Ground states of infinite $q$-deformed Heisenberg ferromagnet Preprint, Osnabrück, 1994

11 Liggett, T M : Interacting Particle Systems Berlin-Heidelberg-New York: Springer, 1981

12 Lindblad, G: On the Generator of Quantum Dynamical Semigroups Commun Math Phys 48, 119-130 (1976)

13 Matsui, T : Markov semigroups on UHF algebras Rev Math Phys 5, 587-600 (1993)

14 Matsui, T : Quantum Statistical Mechanics and Feller Semigroup, Preprint

15 Paulsen, V I : Completely Bounded Maps and Dilations Pitman Research Notes in Mathematics Series 146, London: Longman

16 Richter, S, Werner, R : Ergodicity of Quantum Cellular Automata J Stat Phys 82, 963-998 (1996)

17 Ruelle, D : Thermodynamic Formalism. Reading, MA: Addisson Wesley, 1978

18 Simon, B : Statistical Mechanics of Lattice Gases I Princeton, NJ: Princeton Univ Press, 1993 
